\title{
Minimization Interchange Theorem on Posets
}

\author{
Jean-Philippe Chancelier* Michel De Lara* Benoît Tran* \\ July 14, 2021
}

\begin{abstract}
Interchange theorems between minimization and integration are useful in optimization, especially in optimal control and in stochastic optimization. In this article, we establish a generalized minimization interchange theorem, where integration is replaced by a monotone mapping between posets (partially ordered sets). As an application, we recover, and slightly extend, classical results from the literature, and we tackle the case of the Choquet integral. Our result provides insight on the mechanisms behind existing interchange results.
\end{abstract}

\section{Introduction}

The question of interchanging integration and minimization is an important issue in stochastic optimization (where integration corresponds to mathematical expectation). Let $\overline{\mathbb{R}}=\mathbb{R} \cup\{+\infty\} \cup\{-\infty\}$. Loosely stated, given a measured space $(\Omega, \mathcal{F}, \mu)$ and a subset $X \subset \overline{\mathbb{R}}^{\Omega}$ of functions, an interchange property has the form

$$
\inf _{x \in X} \int_{\Omega} x \mathrm{~d} \mu=\int_{\Omega} \inf _{x \in X} x \mathrm{~d} \mu .
$$

In Equation (1), one needs to clarify in which sense the integral $\int$, the infima $\inf _{X \in X} x$ and $\inf _{x \in X} \int x \mathrm{~d} \mu$ are defined. Mathematical frameworks and conditions to obtain Equation (1) can be found in $[4,5,7,9,13,15]$. We detail

*CERMICS, École des Ponts ParisTech, France 
the contributions of the two references [7] and [13]. We refer the reader to Appendix A for recalls and notation regarding extended Lebesgue and outer integrals.

When the subset $X$, over which minimization is performed, is a subset of $L^{1}(\Omega, \mathcal{F}, \mu ; \mathbb{R})$ and when the integral $\int$ is the usual Lebesgue integral, Giner obtained in [7] a necessary and sufficient condition for (1) as follows. In this case, the space $L^{1}(\Omega, \mathcal{F}, \mu ; \mathbb{R})$ is endowed with the usual $\mu$-pointwise order, and the infimum is $\inf _{x \in X} x=\operatorname{essinf}_{x \in X} x$, which is well-defined by [11, Proposition II.4.1]. Given a subset $X \subset L^{1}(\Omega, \mathcal{F}, \mu ; \mathbb{R})$ of functions, Giner establishes that Equation (1) holds true if and only if, for every finite family $x_{1}, \ldots, x_{n}$ in $X$, we have

$$
\inf _{x \in X} \int_{\Omega}\left(x-\inf _{1 \leq i \leq n} x_{i}\right) \mathrm{d} \mu \leq 0
$$

However, checking the above condition is not an easy task, as it depends jointly on the integral $\int$ and on the subset $X$. Moreover, one may wonder if Equation (1) still holds true for more general subsets $X$, containing functions which are integrable in a weaker sense than Lebesgue integrable.

When a subset $X \subset L^{0}(\Omega, \mathcal{F}, \mu ; \overline{\mathbb{R}})$ of measurable functions is the image of a set $U$ by a mapping $f: L^{0}(\Omega, \mathcal{F}, \mu ; \overline{\mathbb{R}}) \rightarrow L^{0}(\Omega, \mathcal{F}, \mu ; \overline{\mathbb{R}})$, i.e. $X=f(U)$, a celebrated theorem of Rockafellar and Wets ([13, Theorem 14.60]) gives a condition on the mapping $f$ and a condition on the set $U$ so that Equation (1) holds true. In this case, we deal with minimization over subsets $X$ of $L^{0}(\Omega, \mathcal{F}, \mu ; \mathbb{R})$ and interchange with the outer integral, a generalization of the Lebesgue integral to $L^{0}(\Omega, \mathcal{F}, \mu ; \overline{\mathbb{R}})$.

Our contribution is to provide a minimization interchange theorem where integration is replaced by a monotone mapping $\Phi: \mathbb{X} \rightarrow \mathbb{Y}$ between posets (partially ordered sets) $\mathbb{X}$ and $\mathbb{Y}$. More precisely, we provide an abstract interchange theorem of the form

$$
\bigwedge_{x \in X} \Phi(x)=\Phi\left(\bigwedge_{x \in X} x\right)
$$

Several works studied the abstract interchange of Equation (3) with $\Phi$ not being the integral, for instance [1] when $\Phi$ is an $\alpha$-subhomogeneous operator or [14] when $\Phi$ is a risk measure. Once assumed conditions on the mapping $\Phi: \mathbb{X} \rightarrow \mathbb{Y}$ and structural properties of the sets $\mathbb{X}$ and $\mathbb{Y}$, we provide a necessary and sufficient condition so that Equation (3) holds true. Our search for minimal assumptions led us to assume that the sets $\mathbb{X}$ and $\mathbb{Y}$ are equipped 
with partial orders, and that the subset $X \subset \mathbb{X}$ - on which the interchange property is to be checked - is included in a complete inf-semilattice to obtain the existence of greatest lower bound. Our result is in the lineage of Giner's condition (2), as our necessary and sufficient condition involves both the mapping $\Phi$ and the set $X$.

The article is organized as follows. Sect. 2 is devoted to a minimization interchange theorem on posets. Sect. 3 tackles the question of interchange between minimization and different integrations (extended Lebesgue, outer and Choquet integrals), as well as order preserving functionals, by specifying the results of Section 2. We provide background on extended Lebesgue and outer integrals in Appendix A.

We hope that our abstract interchange theorem - together with its application to different integrals and order preserving functionals — provides insight into how one may obtain interchange between minimization and integration, or even go beyond the integral case, like with risk measures in stochastic optimization.

\section{Minimization interchange theorem on posets}

In $\S 2.1$, we present our main result, namely Theorem 2, which provides an abstract interchange result in the form of Equation (3) for a mapping $\Phi: \mathbb{X} \rightarrow$ $\mathbb{Y}$ (generalization of the integral) between specific posets. For this purpose, we define the notion of $\Phi$-inf-directed subset $X \subset \mathbb{X}$, as it is instrumental to obtain necessary and sufficient conditions for such an abstract interchange to hold true. Then, in $\S 2.2$, we show how the well-known notion of inf-directed subset is both sufficient and easier to check for the subset $X \subset \mathbb{X}$ to be $\Phi$-inf-directed.

\subsection{Main result}

Before stating Theorem 2, we provide background on posets and lattices, as well as two new definitions.

We say that $(\mathbb{X}, \preceq)$ is a poset when $\mathbb{X}$ is a set and $\preceq$ is a partial order on $\mathbb{X}$, that is, a reflexive, antisymmetric and transitive binary relation. Examples

include $\mathbb{R}$ or $\overline{\mathbb{R}}$ equipped with the classic order $\leq$, or mappings with values in a poset and equipped with the componentwise order. 
Consider a poset $(\mathbb{X}, \preceq)$ and a subset $X \subset \mathbb{X}$. Any $x^{\prime} \in \mathbb{X}$ such that, for all $x \in X$, we have that $x^{\prime} \preceq x$ is called a lower bound of the set $X$. If a lower bound $x^{\prime} \in \mathbb{X}$ of the set $X$ is such that $x^{\prime \prime} \preceq x^{\prime}$, for any other lower bound $x^{\prime \prime} \in \mathbb{X}$ of the set $X$, then $x^{\prime}$ is unique and is called the greatest lower bound of the set $X$. In that case, it is denoted by inf $X$ or, more explicitly, by $\bigwedge_{x \in X} x$. We say that a poset $(\mathbb{X}, \preceq)$ is an inf-semilattice, if every nonempty finite subset of $\mathbb{X}$ has a greatest lower bound. We say that a poset $(\mathbb{X}, \preceq)$ is a complete inf-semilattice, if every nonempty subset of $\mathbb{X}$ has a greatest lower bound.

Now, we introduce the notion of $\Phi$-inf-directed subset.

Definition 1. Let $\left(\mathbb{X}, \preceq_{\mathbb{X}}\right)$ be an inf-semilattice and $\left(\mathbb{Y}, \preceq_{\mathbb{Y}}\right)$ be a complete inf-semilattice and $\Phi: \mathbb{X} \rightarrow \mathbb{Y}$ be a mapping. Let $X \subset \mathbb{X}$ be a subset of $\mathbb{X}$. We say that the subset $X$ is $\Phi$-inf-directed if, for every finite subset $\tilde{X} \subset X$, we have that

$$
\bigwedge_{x \in X} \Phi(x) \preceq \mathbb{Y} \Phi\left(\bigwedge_{x \in \tilde{X}} x\right)
$$

With this definition, we can now state our main theorem.

Theorem 2 (Minimization Interchange Theorem). Let $(\mathbb{X}, \preceq \mathbb{X})$ be a poset and $\left(\mathbb{Y}, \preceq_{\mathbb{Y}}\right)$ be a complete inf-semilattice. Let $X \subset \mathbb{X}$ be a subset of $\mathbb{X}$, $\widetilde{\mathbb{X}} \subset \mathbb{X}$ be an inf-semilattice such that $X \subset \widetilde{\mathbb{X}} \subset \mathbb{X}$, and $\Phi: \mathbb{X} \rightarrow \mathbb{Y}$ be a mapping such that

$\left(T_{1}\right)$ the mapping $\Phi$ is order preserving, i.e. for every $x, x^{\prime} \in \mathbb{X}$,

$$
x \preceq_{\mathbb{X}} x^{\prime} \Rightarrow \Phi(x) \preceq_{\mathbb{Y}} \Phi\left(x^{\prime}\right),
$$

$\left(T_{2}\right)$ The element $\wedge_{x \in X} x$ exists in the poset $\mathbb{X}$ and there exists a sequence $\left\{x_{n}\right\}_{n \in \mathbb{N}}$ in $X$ such that

(a) $\wedge_{n \in \mathbb{N}} x_{n}$ exists in $\mathbb{X}$ and

$$
\bigwedge_{n \in \mathbb{N}} x_{n}=\bigwedge_{x \in X} x
$$

(b) the nonincreasing sequence $\left\{x_{n}^{\prime}\right\}_{n \in \mathbb{N}}$ in $\widetilde{\mathbb{X}}$ defined by $x_{n}^{\prime}=\wedge_{k \leq n} x_{k}$, for all $n \in \mathbb{N}$, satisfies the following inequality

$$
\bigwedge_{n \in \mathbb{N}} \Phi\left(x_{n}^{\prime}\right) \preceq_{\mathbb{Y}} \Phi\left(\bigwedge_{n \in \mathbb{N}} x_{n}\right)=\Phi\left(\bigwedge_{x \in X} x\right) .
$$


Then, we have the interchange formula

$$
\bigwedge_{x \in X} \Phi(x)=\Phi\left(\bigwedge_{x \in X} x\right)
$$

if and only if the subset $X$ is $\Phi$-inf-directed (as in Definition 1).

Proof. Let $\Phi: \mathbb{X} \rightarrow \mathbb{Y}$ and $X \subset \mathbb{X}$ be given satisfying the two assumptions $\left(T_{1}\right)$ and $\left(T_{2}\right)$.

- We assume that the subset $X$ is $\Phi$-inf-directed and we prove the interchange formula (8) by means of two inequalities.

First, using the fact that the mapping $\Phi$ is order preserving, we have that

$$
\Phi\left(\wedge_{x \in X} x\right) \preceq_{\mathbb{Y}} \Phi\left(x^{\prime}\right), \quad \forall x^{\prime} \in X,
$$

where $\wedge_{x \in X} x$ is well-defined as an element of $\mathbb{X}$ by Assumption $\left(T_{2}\right)$ Thus, by the assumption that $\left(\mathbb{Y}, \preceq_{\mathbb{Y}}\right)$ is a complete inf-semilattice, we obtain that

$$
\Phi(\hat{x \in X} x) \preceq_{\mathbb{Y}} \underset{x \in X}{\wedge} \Phi(x) .
$$

Second, we prove the reverse inequality $\wedge_{x \in X} \Phi(x) \preceq_{\mathbb{Y}} \Phi\left(\wedge_{x \in X} x\right)$. Using Assumption $\left(T_{2}\right)$, there exists a sequence $\left\{x_{n}\right\}_{n \in \mathbb{N}}$, whose terms are in $X$ (hence in the inf-semilattice $\widetilde{\mathbb{X}}$ ), and such that $\wedge_{n \in \mathbb{N}} x_{n}=\wedge_{x \in X} x$ by (6). Now, we define a new sequence $\left\{x_{n}^{\prime}\right\}_{n \in \mathbb{N}}$ by $x_{n}^{\prime}=\wedge_{k \leq n} x_{k}$, for all $n \in \mathbb{N}$. So defined, $x_{n}^{\prime}$ does not necessarily belong to the subset $X$, but belongs to the inf-semilattice $\widetilde{\mathbb{X}}$ which contains $X$. Then, we get

$$
\bigwedge_{x \in X} \Phi(x) \preceq_{\mathbb{Y}} \Phi\left(\bigwedge_{k \leq n} x_{k}\right)
$$

by (4) as the subset $X$ is $\Phi$-inf-directed, by assumption, and as the set $\left\{x_{k} \mid k \leq n\right\} \subset X$ is finite

$$
=\Phi\left(x_{n}^{\prime}\right)
$$

by definition of $x_{n}^{\prime}=\bigwedge_{k \leq n} x_{k}$, so that we deduce

$$
\bigwedge_{x \in X} \Phi(x) \preceq_{\mathbb{Y}} \bigwedge_{n \in \mathbb{N}} \Phi\left(x_{n}^{\prime}\right)
$$

(as $\left(\mathbb{Y}, \preceq_{\mathbb{Y}}\right.$ ) is a complete inf-semilattice by assumption)

$$
\preceq_{\mathbb{Y}} \Phi\left(\bigwedge_{n \in \mathbb{N}} x_{n}\right)=\Phi\left(\bigwedge_{x \in X} x\right)
$$


by (7) Assumption $\left(T_{2}\right)$ is satisfied.

- Conversely, we assume that the interchange formula (8) holds true for the subset $X \subset \mathbb{X}$, and we show that $X$ is $\Phi$-inf-directed.

For this purpose, we consider a finite subset $\tilde{X} \subset X$, and we get

$$
\begin{aligned}
\bigwedge_{x \in X} \Phi(x) & =\Phi\left(\bigwedge_{x \in X} x\right) \quad \text { (by the interchange formula (8)) } \\
& \preceq_{\mathbb{Y}} \Phi\left(\bigwedge_{x \in \tilde{X}} x\right)
\end{aligned}
$$

since the mapping $\Phi$ is order preserving and $\wedge_{x \in X} x \preceq_{\mathbb{X}} \wedge_{x \in \tilde{X}} x$.

This concludes the proof.

To state a corollary, we introduce the notion of sequentially-inf continuity of a mapping. The name is suggested by the notion of sequentially order continuity (denoted as "continuité monotone séquentielle" in [11, p. 37]).

Definition 3. Let $\left(\mathbb{X}, \preceq_{\mathbb{X}}\right)$ be an inf-semilattice and $\left(\mathbb{Y}, \preceq_{\mathbb{Y}}\right)$ be a complete inf-semilattice, $\Phi: \mathbb{X} \rightarrow \mathbb{Y}$ be a mapping and $\underline{x}$ be a given point of $\mathbb{X}$. We say that the mapping $\Phi$ is sequentially-inf continuous at $\underline{x}$ when the following property holds true: for any nonincreasing sequence $\left\{x_{n}\right\}_{n \in \mathbb{N}}$ in $\mathbb{X}$ such that $\bigwedge_{n \in \mathbb{N}} x_{n}$ exists (in $\mathbb{X}$ ) and such that $\bigwedge_{n \in \mathbb{N}} x_{n}=\underline{x}$, we have that

$$
\bigwedge_{n \in \mathbb{N}} \Phi\left(x_{n}\right) \preceq_{\mathbb{Y}} \Phi\left(\bigwedge_{n \in \mathbb{N}} x_{n}\right)=\Phi(\underline{x}) .
$$

Moreover, we say that the mapping $\Phi$ is sequentially-inf continuous on the inf-semilattice $\mathbb{X}$ if it is sequentially-inf continuous at every $x \in \mathbb{X}$.

This definition is demanding as the inequality in (9) is the reverse of the inequality obtained when the mapping $\Phi$ is nondecreasing, or the inequality given by the Fatou Lemma when the mapping $\Phi$ is the Lebesgue integral, or the inequality required if the mapping $\Phi$ is lower semi continuous.

Corollary 4 (Minimization Interchange Corollary). Let $\left(\mathbb{X}, \preceq_{\mathbb{X}}\right)$ be a poset and $\left(\mathbb{Y}, \preceq_{\mathbb{Y}}\right)$ be a complete inf-semilattice. Let $X \subset \mathbb{X}$ be a subset of $\mathbb{X}$, $\widetilde{\mathbb{X}} \subset \mathbb{X}$ be an inf-semilattice such that $X \subset \widetilde{\mathbb{X}} \subset \mathbb{X}$, and $\Phi: \mathbb{X} \rightarrow \mathbb{Y}$ be a mapping such that

$\left(C_{1}\right)$ the mapping $\Phi$ is order preserving, i.e. for every $x, x^{\prime} \in \mathbb{X}$,

$$
x \preceq_{\mathbb{X}} x^{\prime} \Rightarrow \Phi(x) \preceq_{\mathbb{Y}} \Phi\left(x^{\prime}\right),
$$


$\left(C_{2}\right)$ the subset $X$ has the countable inf property, i.e. $\wedge_{x \in X} x$ exists in the poset $\mathbb{X}$ and there exists a sequence $\left\{x_{n}\right\}_{n \in \mathbb{N}}$ in $X$ such that $\wedge_{n \in \mathbb{N}} x_{n}$ exists in $\mathbb{X}$ and

$$
\bigwedge_{n \in \mathbb{N}} x_{n}=\bigwedge_{x \in X} x,
$$

$\left(C_{3}\right)$ the mapping $\Phi$ is sequentially-inf continuous at $\underline{x}=\wedge_{x \in X} x$, when restricted to the inf-semilattice $\widetilde{\mathbb{X}}$ (see Definition 3).

Then, we have the interchange formula

$$
\bigwedge_{x \in X} \Phi(x)=\Phi\left(\bigwedge_{x \in X} x\right)
$$

if and only if the subset $X$ is $\Phi$-inf-directed (as in Definition 1).

Proof. Let $\Phi: \mathbb{X} \rightarrow \mathbb{Y}$ and $X \subset \mathbb{X}$ be given satisfying the three assumptions $\left(C_{1}\right),\left(C_{2}\right)$, and $\left(C_{3}\right)$ of Corollary 4 . We are going to show that the two assumptions $\left(T_{1}\right)$ and $\left(T_{2}\right)$ of Theorem 2 are satisfied. There is nothing to show for assumptions $\left(C_{1}\right)$ and $\left(T_{1}\right)$ that coincide.

Then, as $\left(C_{2}\right)$ and $\left(C_{3}\right)$ hold true, from the sequence $\left\{x_{n}\right\}_{n \in \mathbb{N}}$ given by $\left(C_{2}\right)$, we build the nonincreasing sequence $\left\{x_{n}^{\prime}\right\}_{n \in \mathbb{N}}$ given by $x_{n}^{\prime}=\bigwedge_{k \leq n} x_{k}$, for all $n \in \mathbb{N}$. As $\wedge_{n \in \mathbb{N}} x_{n}=\wedge_{x \in X} x$ by $\left(C_{2}\right)$, it readily follows that $\bigwedge_{x \in X} x \preceq_{\mathbb{X}} x_{n}^{\prime} \preceq_{\mathbb{X}} x_{n}$, for all $n \in \mathbb{N}$. We deduce that $\bigwedge_{n \in \mathbb{N}} x_{n}^{\prime}=\bigwedge_{x \in X} x$. Indeed, $\wedge_{x \in X} x$ is a minorant of the set $\left\{x_{n}^{\prime} \mid n \in \mathbb{N}\right\}$ and, if there were a strictly greater minorant, it would be a strictly greater minorant also for the set $\left\{x_{n} \mid n \in \mathbb{N}\right\}$, which is not the case as $\wedge_{n \in \mathbb{N}} x_{n}=\bigwedge_{x \in X} x$ by assumption. As a consequence, the sequence $\left\{x_{n}^{\prime}\right\}_{n \in \mathbb{N}}$ (whose terms do not necessarily belong to the subset $X$, whereas those of the sequence $\left\{x_{n}\right\}_{n \in \mathbb{N}}$ do) is nonincreasing and satisfies the equalities $\wedge_{n \in \mathbb{N}} x_{n}^{\prime}=\wedge_{n \in \mathbb{N}} x_{n}=\bigwedge_{x \in X} x$. Thus, we have shown (6), which represents half of Assumption $\left(T_{2}\right)$ of Theorem 2.

To prove (7), the second half of Assumption $\left(T_{2}\right)$ of Theorem 2, we simply use Definition 3. Indeed, Equation (9) with the nonincreasing sequence $\left\{x_{n}^{\prime}\right\}_{n \in \mathbb{N}}$, which satisfies $\wedge_{n \in \mathbb{N}} x_{n}^{\prime}=\wedge_{x \in X} x=\underline{x}$, gives $\wedge_{n \in \mathbb{N}} \Phi\left(x_{n}^{\prime}\right) \preceq_{\mathbb{Y}}$ $\Phi\left(\wedge_{n \in \mathbb{N}} x_{n}^{\prime}\right)$, from which we readily get (7) as we have shown that $\wedge_{n \in \mathbb{N}} x_{n}^{\prime}=$ $\bigwedge_{n \in \mathbb{N}} x_{n}=\bigwedge_{x \in X} x$.

This ends the proof. 


\subsection{A sufficient condition for $\Phi$-directed sets}

Given an order preserving and sequentially-inf-continuous mapping $\Phi: \mathbb{X} \rightarrow$ $\mathbb{Y}$, where the posets $\mathbb{X}, \mathbb{Y}$ have sufficient structure, the Minimization Interchange Theorem 2 shows that a subset $X \in \mathbb{X}$ is $\Phi$-inf-directed if, and only if, we have the abstract interchange formula $\wedge_{x \in X} \Phi(x)=\Phi\left(\wedge_{x \in X} x\right)$. However, as made apparent in its name, checking if a subset $X$ is $\Phi$-inf-directed is a condition that involves both $X$ and its image by the mapping $\Phi$. We give a simple sufficient condition on the subset $X$ only which ensures that $X$ is $\Phi$-inf-directed for any order preserving mapping $\Phi$.

Let $(\mathbb{X}, \preceq)$ be a poset. An inf-directed ${ }^{1}$ set $X \subset \mathbb{X}$ is a nonempty set with the property that, for every $x, x^{\prime} \in X$, there exists $x^{\prime \prime} \in X$ such that $x^{\prime \prime} \preceq x$ and $x^{\prime \prime} \preceq x^{\prime}$.

We now prove in Lemma 5 that any inf-directed subset $X \subset \mathbb{X}$ is $\Phi$-infdirected for any order preserving mapping $\Phi$.

Lemma 5 (Inf-directed implies $\Phi$-inf-directed). Let $\left(\mathbb{X}, \preceq_{\mathbb{X}}\right)$ be an inf-semilattice, $X \subset \mathbb{X}$ be a subset, and $\left(\mathbb{Y}, \preceq_{\mathbb{Y}}\right)$ be a complete inf-semilattice. If the subset $X$ is inf-directed then $X$ is $\Phi$-inf-directed for any order preserving mapping $\Phi:\left(\mathbb{X}, \preceq_{\mathbb{X}}\right) \rightarrow\left(\mathbb{Y}, \preceq_{\mathbb{Y}}\right)$.

Proof. Suppose that $X \subset \mathbb{X}$ is an inf-directed subset of $(\mathbb{X}, \preceq)$, and let $\Phi$ : $\left(\mathbb{X}, \preceq_{\mathbb{X}}\right) \rightarrow\left(\mathbb{Y}, \preceq_{\mathbb{Y}}\right)$ be an order preserving mapping. We prove that the subset $X$ is $\Phi$-inf-directed.

For this purpose, we consider a finite subset $\tilde{X} \subset X$. Then, by repeated application of the inf-directed property to the finite number of elements in the subset $\tilde{X}$, we get that there exists $\tilde{x} \in X$ such that $\tilde{x} \preceq_{\mathbb{X}} \wedge_{x \in \tilde{X}} x$. We therefore obtain that

$$
\begin{array}{rrr}
\bigwedge_{x \in X} \Phi(x) & \preceq_{\mathbb{Y}} \Phi(\tilde{x}) & (\text { as } \tilde{x} \in X) \\
\preceq_{\mathbb{Y}} \Phi\left(\wedge_{x \in \tilde{X}} x\right), \quad\left(\text { as } \Phi \text { is order preserving and } \tilde{x} \preceq_{\mathbb{X}} \wedge_{x \in \tilde{X}} x\right)
\end{array}
$$

which ensures that $X$ is $\Phi$-inf-directed and concludes the proof.

The converse is false, i.e. $\Phi$-inf-directed subsets are not necessarily infdirected subsets as detailed now in Example 1.

\footnotetext{
${ }^{1}$ It is also called a filtered set $[6]$.
} 
Example 1 (The converse of Lemma 5 is false). Consider $\Omega=\mathbb{R}$ equipped with its Borel $\sigma$-algebra $\mathcal{B}(\mathbb{R})$ and Lebesgue measure $\lambda$. Define the infsemilattice $\mathbb{X}=L_{\oplus}^{1}(\Omega, \mathcal{F}, \mu ; \overline{\mathbb{R}})$ (the set of measurable functions with Lebesgue integrable positive part, see Appendix A) with the $\mu$-pointwise order and the mapping $\Phi: \mathbb{X} \rightarrow \overline{\mathbb{R}}$ being the (extended) Lebesgue integral. We claim that the subset $X \subset \mathbb{X}$, defined by $X=\left(-n \mathbf{1}_{(n, n+1)}, n \in \mathbb{N}\right) \subset L_{\oplus}^{1}(\Omega, \mathcal{F}, \mu ; \overline{\mathbb{R}})$, is $\Phi$-inf-directed but not inf-directed.

First, we calculate $\wedge_{x \in X} \Phi(x)=\wedge_{x \in X} \int_{\mathbb{R}} x(y) \lambda(\mathrm{d} y)=\wedge_{n \in \mathbb{N}}(-n)=-\infty$. Second, for every finite subset $\tilde{X}=\left\{x_{n_{1}}, \ldots, x_{n_{k}}\right\} \subset X$ of functions, we have that $-k \max _{1 \leq i \leq k} n_{i} \leq \Phi\left(\wedge_{x \in \tilde{X}} x\right)$. Thus, we get that

$$
\wedge_{x \in X} \Phi(x)=-\infty \leq-k \max _{1 \leq i \leq k} n_{i} \leq \Phi\left(\wedge_{x \in \tilde{X}} x\right)
$$

hence the subset $X$ is $\Phi$-inf-directed.

Nevertheless, $X$ is not an inf-directed subset of $(\mathbb{X}, \preceq)$. Indeed, let, for all $k \in \mathbb{N}$, the function $\psi_{k}$ be defined by $\psi_{k}=-k \mathbf{1}_{(k, k+1)}$, and let $n$ and $n^{\prime}$ in $\mathbb{N}$ be fixed such that $n \neq n^{\prime}$. Assume that there exists $n^{\prime \prime} \in \mathbb{N}$ such that $\psi_{n^{\prime \prime}} \leq \psi_{n} \wedge \psi_{n^{\prime}}$. Then, if $X$ were an inf-directed subset of $(\mathbb{X}, \preceq)$, we should have, using the definition of the functions $\left\{\psi_{k}\right\}_{k \in \mathbb{N}}$, that the support of $\psi_{n^{\prime \prime}}$ should contain the set $(n, n+1) \cup\left(n^{\prime}, n^{\prime}+1\right)$. However no function of $X$ has for support the union of two such intervals of unit length.

In this case, we can observe that the interchange between integration and minimization holds true. Indeed, on the one hand we have shown above that $\wedge_{x \in X} \Phi(x)=-\infty$ and, on the other hand, we have that

$$
\Phi\left(\wedge_{x \in X} x\right) \leq \int_{0}^{+\infty}(1-y) \lambda(\mathrm{d} y)=-\infty
$$

hence that $\wedge_{x \in X} \Phi(x)=-\infty=\Phi\left(\wedge_{x \in X} x\right)$.

\section{Applications to minimization on functional spaces}

This section is devoted to applications of the Minimization Interchange Theorem 2 (and its Corollary 4) to the case of interchange between (an extension of) the Lebesgue integral and minimization for suitable subsets of measurable functions. 
In $\S 3.1$, we treat the case of interchange between minimization and integration, and we recover both interchange theorems of Giner and RockafellarWets. In $\S 3.2$, we recover an interchange theorem of Shapiro for order preserving functionals. Lastly, in $§ 3.3$, we study the case of the Choquet integral.

\subsection{Interchange between minimization and integration}

We consider a measured space $(\Omega, \mathcal{F}, \mu)$. We refer the reader to Appendix A for material regarding extended Lebesgue and outer integrals. In §3.1.1, we apply the abstract results of Section 2 to the case of suitable subsets of measurable functions, and obtain a new Theorem 6. In $\S 3.1 .2$ and in $\S 3.1 .3$, we recover the interchange theorems of Giner and Rockafellar-Wets from Theorem 6.

\subsubsection{Main result with integrals}

We apply the abstract results of Section 2 to the case of subsets of $\mathbb{X}=$ $L_{\oplus}^{1}(\Omega, \mathcal{F}, \mu ; \overline{\mathbb{R}})$, the set of measurable functions with Lebesgue integrable positive $^{2}$ part. We consider the interchange with the mapping $\Phi: \mathbb{X} \rightarrow \overline{\mathbb{R}}$ being the extended Lebesgue integral on $\mathbb{X}$.

We state the main result about the interchange between the extended Lebesgue integral $\int_{\Omega}: L_{\oplus}^{1}(\Omega, \mathcal{F}, \mu ; \overline{\mathbb{R}}) \rightarrow \overline{\mathbb{R}}$ and minimization.

Theorem 6. Let $X$ be a subset of $L_{\oplus}^{1}(\Omega, \mathcal{F}, \mu ; \overline{\mathbb{R}})$. Then, $\operatorname{essinf}_{x \in X} x \in$ $L_{\oplus}^{1}(\Omega, \mathcal{F}, \mu ; \overline{\mathbb{R}})$ and the following equality

$$
\inf _{x \in X} \int_{\Omega} x \mathrm{~d} \mu=\int_{\Omega} \underset{x \in X}{\operatorname{essinf}} x \mathrm{~d} \mu
$$

is valid if an only if $X$ is integrably inf-directed, i.e. for every finite family $x_{1}, \ldots, x_{n}$ in $X$ we have

$$
\inf _{x \in X} \int_{\Omega} x \mathrm{~d} \mu \leq \int_{\Omega} \inf _{1 \leq i \leq n} x_{i} \mathrm{~d} \mu
$$

Proof. As being integrably inf-directed defined here coincides with being $\Phi$ inf-directed (see Definition 1) when $\Phi=\int_{\Omega}$ is the extended Lebesgue integral

\footnotetext{
${ }^{2}$ Mutatis mutandis, we could as well consider $\mathbb{X}=L_{\ominus}^{1}(\Omega, \mathcal{F}, \mu ; \overline{\mathbb{R}})$, the set of measurable functions with Lebesgue integrable negative part and maximization in lieu of minimization.
} 
on $L_{\oplus}^{1}(\Omega, \mathcal{F}, \mu ; \overline{\mathbb{R}})$, we will show that the assumptions of Corollary 4 are fulfilled to obtain Theorem 6 as a special case.

The proof is broken into two parts. First, the assumptions of Corollary 4 are satisfied by Proposition 7. Namely, the structural assumptions on the domain of $\Phi: L_{\oplus}^{1}(\Omega, \mathcal{F}, \mu ; \overline{\mathbb{R}}) \rightarrow \overline{\mathbb{R}}$ are satisfied (see $\S 2.1$ for recalls on the notions below):

- The set $\mathbb{X}=\widetilde{\mathbb{X}}=L^{0}(\Omega, \mathcal{F}, \mu ; \overline{\mathbb{R}})$ with the $\mu$-pointwise order is a complete inf-semilattice;

- Every subset $X \subset \widetilde{\mathbb{X}}=\mathbb{X}$ has the countable inf property.

Moreover, $\mathbb{Y}=\overline{\mathbb{R}}$ with the usual order is a complete inf-semilattice. Second, by Proposition 8 , the extended Lebesgue integral $\int_{\Omega}: L_{\oplus}^{1}(\Omega, \mathcal{F}, \mu ; \overline{\mathbb{R}}) \rightarrow \overline{\mathbb{R}}$ is order preserving and sequentially-inf continuous.

This ends the proof.

Note that, as semi-integrable functions - that is, measurable functions with either Lebesgue integrable positive part or Lebesgue integrable negative part — are linked by the relation (see Lemma 14) $x \in L_{\ominus}^{1}(\Omega, \mathcal{F}, \mu ; \overline{\mathbb{R}}) \Leftrightarrow-x \in$ $L_{\oplus}^{1}(\Omega, \mathcal{F}, \mu ; \overline{\mathbb{R}})$, one can deduce a symmetric result about the interchange between extended Lebesgue integral and maximization.

We check in Proposition 7 (structural properties of the spaces of measurable and semi-integrable functions) and Proposition 8 (properties of the outer integral) that the assumptions of the Minimization Interchange Corollary 4 are satisfied.

Proposition 7 (Structural properties of the space of measurable and semi-integrable functions $)$. The set $L^{0}(\Omega, \mathcal{F}, \mu ; \overline{\mathbb{R}})$ and its subset $L_{\oplus}^{1}(\Omega, \mathcal{F}, \mu ; \overline{\mathbb{R}})$, both equipped with the $\mu$-pointwise order, are complete inf-semilattice with the countable inf property.

Proof.

- We consider the set $L^{0}(\Omega, \mathcal{F}, \mu ; \overline{\mathbb{R}})$. First, the fact that it is a complete inf-semilattice is a consequence of the existence of the essential essential infimum for any family (countable or not) of class of random variables as proved in [11, Proposition II.4.1] (the proof is for probability measures but it extends easily to $\sigma$-finite measures). We rephrase here the existence result of [11, Proposition II.4.1]. For any class family (countable or not) $\left\{x_{i}\right\}_{i \in I}$ in $L^{0}(\Omega, \mathcal{F}, \mu ; \overline{\mathbb{R}})$, there exists a unique class ess $\inf _{i \in I} x_{i} \in L^{0}(\Omega, \mathcal{F}, \mu ; \overline{\mathbb{R}})$ which 
is a greatest lower bound of the family $\left\{x_{i}\right\}_{i \in I}$. That is, for any function $\underline{x} \in L^{0}(\Omega, \mathcal{F}, \mu ; \overline{\mathbb{R}})$, we have

$$
\forall i \in I, \underline{x} \leq x_{i} \Leftrightarrow \underline{x} \leq \underset{i \in I}{\operatorname{essinf}} x_{i}
$$

The fact that there exists a countable subfamily $\left\{x_{i_{n}}\right\}_{n \in \mathbb{N}}$ such that

$$
\underset{i \in I}{\operatorname{essinf}} x_{i}=\inf _{n \in \mathbb{N}} x_{i_{n}}
$$

is not stated explicitly in [11, Proposition II.4.1], but it is stated in the proof as an intermediate result to obtain the essential infimum. It is immediate that the countable subfamily can be chosen as a nonincreasing sequence, a property that will be useful right below.

- We consider the set $L_{\oplus}^{1}(\Omega, \mathcal{F}, \mu ; \overline{\mathbb{R}})$ and consider a class family (countable or not) $\left\{u_{i}\right\}_{i \in I}$ in $L_{\oplus}^{1}(\Omega, \mathcal{F}, \mu ; \overline{\mathbb{R}})$. As $L_{\oplus}^{1}(\Omega, \mathcal{F}, \mu ; \overline{\mathbb{R}})$ is a subset of $L^{0}(\Omega, \mathcal{F}, \mu ; \overline{\mathbb{R}})$ we obtain (using the first part of the proof) the existence of $\operatorname{ess}_{i \in I} u_{i} \in L^{0}(\Omega, \mathcal{F}, \mu ; \overline{\mathbb{R}})$ and the existence of a nonincreasing countable subfamily $\left\{u_{i_{n}}\right\}_{n \in \mathbb{N}}$ such that

$$
\underset{i \in I}{\operatorname{essinf}} u_{i}=\inf _{n \in \mathbb{N}} u_{i_{n}}
$$

Using the monotone convergence theorem for $L_{\oplus}^{1}(\Omega, \mathcal{F}, \mu ; \overline{\mathbb{R}}$ ) (see Proposition 15 in Appendix A), we obtain that $\operatorname{essinf}_{i \in I} u_{i} \in L_{\oplus}^{1}(\Omega, \mathcal{F}, \mu ; \overline{\mathbb{R}})$, as the infimum of a sequence in $L_{\oplus}^{1}(\Omega, \mathcal{F}, \mu ; \overline{\mathbb{R}})$. As a consequence, the subset $L_{\oplus}^{1}(\Omega, \mathcal{F}, \mu ; \overline{\mathbb{R}})$ is a complete inf-semilattice which has the countable inf property.

This ends the proof.

Proposition 8 (Properties of the outer and extended Lebesgue integrals).

- The outer integral (32a) is an order preserving mapping between the posets $L^{0}(\Omega, \mathcal{F}, \mu ; \overline{\mathbb{R}})$ and $\overline{\mathbb{R}}$.

- The extended Lebesgue integral (27b) is both order preserving and sequentially-inf continuous on the inf-semilattice $L_{\oplus}^{1}(\Omega, \mathcal{F}, \mu ; \overline{\mathbb{R}})$.

Proof. Following Definition 16 the outer integral is clearly order preserving between $L^{0}(\Omega, \mathcal{F}, \mu ; \overline{\mathbb{R}})$ and $\overline{\mathbb{R}}$. From Proposition 17 , both outer and extended 
Lebesgue integrals coincide on $L_{\oplus}^{1}(\Omega, \mathcal{F}, \mu ; \overline{\mathbb{R}}) \subset L^{0}(\Omega, \mathcal{F}, \mu ; \overline{\mathbb{R}})$, thus the extended Lebesgue integral is also order preserving between $L_{\oplus}^{1}(\Omega, \mathcal{F}, \mu ; \overline{\mathbb{R}})$ and $\overline{\mathbb{R}}$. We prove that the extended Lebesgue integral is sequentially-inf continuous on $L_{\oplus}^{1}(\Omega, \mathcal{F}, \mu ; \overline{\mathbb{R}})$ using the extended monotone convergence Theorem 15 . Let $\left(f_{n}\right)_{n \in \mathbb{N}}$ be an nonincreasing sequence of functions in $L_{\oplus}^{1}(\Omega, \mathcal{F}, \mu ; \overline{\mathbb{R}})$. We put $f=\bigwedge_{n \in \mathbb{N}} f_{n}$, which belongs to the complete inf-semilattice $L^{0}(\Omega, \mathcal{F}, \mu ; \overline{\mathbb{R}})$. By Proposition 15, we get that $f \in L_{\oplus}^{1}(\Omega, \mathcal{F}, \mu ; \overline{\mathbb{R}})$ and that $\wedge_{n \in \mathbb{N}} \int f_{n} d \mu=$ $\int f d \mu$ by (30). Thus, the outer integral (32a) is sequentially-inf continuous on the inf-semilattice $L_{\oplus}^{1}(\Omega, \mathcal{F}, \mu ; \overline{\mathbb{R}})$.

\subsubsection{Comparison with Giner [7]}

From Theorem 6, we now recover the interchange theorem of Giner.

Theorem 9. ([7, Theorem 4.2]) Let $X$ be a subset of $L^{1}(\Omega, \mathcal{F}, \mu ; \mathbb{R})$. The following equality

$$
\inf _{x \in X} \int_{\Omega} x \mathrm{~d} \mu=\int_{\Omega} \operatorname{essinf}_{x \in X} x \mathrm{~d} \mu,
$$

is valid if an only if $X$ is integrably inf-directed, i.e. for any finite family $x_{1}, \ldots, x_{n}$ in $X$ we have

$$
\inf _{x \in X} \int_{\Omega}\left(x-\inf _{1 \leq i \leq n} x_{i}\right) \mathrm{d} \mu \leq 0 .
$$

As $L^{1}(\Omega, \mathcal{F}, \mu ; \mathbb{R}) \subset L_{\oplus}^{1}(\Omega, \mathcal{F}, \mu ; \overline{\mathbb{R}})$, the interchange formula in Theorem 6 is a slight generalization to $L_{\oplus}^{1}(\Omega, \mathcal{F}, \mu ; \overline{\mathbb{R}})$ of Giner's Theorem 9 stated for subsets of $L^{1}(\Omega, \mathcal{F}, \mu ; \mathbb{R})$. This is no surprise, as we are indebted to Giner since Theorem 6 was greatly inspired by Giner's result.

\subsubsection{Comparison with Rockafellar and Wets [13]}

We prove that the Rockafellar-Wets interchange theorem below can be deduced from Theorem 6 combined with [7, Theorem 3.1].

Let $(\Omega, \mathcal{F}, \mu)$ be a measured space with $\mu$ being a $\sigma$-finite measure. As we work with subsets of measurable functions, the integral used here is the outer integral $\int_{\Omega}^{*}$ (see Definition 16 in Appendix A). Following [13], a subset $\mathcal{U} \subset L^{0}\left(\Omega, \mathcal{F}, \mu ; \mathbb{R}^{d}\right)$ is said to be Rockafellar-Wets decomposable (w.r.t. the $\sigma$-finite measure $\mu$ ) if

$y \mathbf{1}_{A}+\mathfrak{u} \mathbf{1}_{A^{c}} \in \mathcal{U}, \quad \forall y \in L^{\infty}\left(A, \mathcal{F}, \mu ; \mathbb{R}^{d}\right), \forall A \in \mathcal{F}, \mu(A)<+\infty, \forall \mathfrak{u} \in \mathcal{U}$. 
The notion of decomposable subsets is widely used in $L^{p}$ spaces and we refer the reader to [7] for a survey on various related definitions.

Theorem 10. ([13, Theorem 14.60]) Let $\mathcal{U}$ be a subset of $L^{0}\left(\Omega, \mathcal{F}, \mu ; \mathbb{R}^{d}\right)$ that is Rockafellar-Wets decomposable. Let $g: \Omega \times \mathbb{R}^{d} \rightarrow \overline{\mathbb{R}}$ be a normal integrand ${ }^{3}$. If there exists $\overline{\mathfrak{u}} \in \mathcal{U}$ such that $g(\cdot, \bar{u}(\cdot)) \in L_{\oplus}^{1}\left(\Omega, \mathcal{F}, \mu ; \mathbb{R}^{d}\right)$, one has that

$$
\inf _{\mathfrak{u} \in \mathcal{U}} \int_{\Omega}^{*} g(\omega, \mathfrak{u}(\omega)) \mathrm{d} \mu(\omega)=\int_{\Omega}^{*}\left(\inf _{u \in \mathbb{R}^{n}} g(\omega, u)\right) \mathrm{d} \mu(\omega)
$$

Moreover, as long as this common value is not $-\infty$, one has that

$\underline{\mathfrak{u}} \in \underset{\mathfrak{u} \in \mathcal{U}}{\arg \min } \int_{\Omega}^{*} g(\omega, \mathfrak{u}(\omega)) \mathrm{d} \mu(\omega) \Longleftrightarrow \underline{\mathfrak{u}} \in \mathcal{U} \quad$ and $\underline{\mathfrak{u}}(\cdot) \in \underset{u \in \mathbb{R}^{n}}{\arg \min } g(\cdot, u) \mu$-a.s. .

The proof relies on the property that the image by a measurable mapping of a Rockafellar-Wets decomposable subset is an integrably inf-directed subset of $L^{0}(\Omega, \mathcal{F}, \mu ; \overline{\mathbb{R}})$.

Proof. (Equation (18) as a consequence of [7, Theorem 3.1] and the Minimization Interchange Corollary 4)

- We introduce the set $X=\{\Omega \ni \omega \mapsto g(\omega, \mathfrak{u}(\omega)) \mid \mathfrak{u} \in \mathcal{U}\}$. Using the fact that the function $g$ is a normal integrand and that $\mathcal{U}$ is a subset of $L^{0}\left(\Omega, \mathcal{F}, \mu ; \mathbb{R}^{d}\right)$, we obtain that $X$ is a subset of $L^{0}(\Omega, \mathcal{F}, \mu ; \overline{\mathbb{R}})[13$, Theorem 14.37] and we can write

$$
\inf _{\mathfrak{u} \in \mathcal{U}} \int_{\Omega}^{*} g(\omega, \mathfrak{u}(\omega)) \mathrm{d} \mu(\omega)=\bigwedge_{x \in X} \int_{\Omega}^{*} x(\omega) \mathrm{d} \mu(\omega)
$$

Now, using the definition (32a) of the outer integral, we have that

$$
\bigwedge_{x \in X} \int_{\Omega}^{*} x(\omega) \mathrm{d} \mu(\omega)=\bigwedge_{x \in X} \inf _{\substack{x^{\prime} \in L^{1}(\Omega, \mathcal{F}, \mu ; \mathbb{R}) \\ x^{\prime} \geq x}} \int_{\Omega} x^{\prime}(\omega) \mathrm{d} \mu(\omega) .
$$

We define the upper set $\underset{(1)}{\uparrow} X$ of $X$ in $L^{1}(\Omega, \mathcal{F}, \mu ; \mathbb{R})$ by

$$
\underset{(1)}{\uparrow} X=\left\{x^{\prime} \in L^{1}(\Omega, \mathcal{F}, \mu ; \mathbb{R}) \mid \exists x \in X \text { s.t. } x \leq x^{\prime} \quad \mu \text {-a.s. }\right\} .
$$

\footnotetext{
${ }^{3}$ See [13, Definition 14.27].
} 
The set ${ }_{(1)}^{\uparrow} X$ is not empty. Indeed, by assumption there exists $\overline{\mathfrak{u}} \in \mathcal{U}$ such that $\bar{x}=g(\cdot, \overline{\mathfrak{u}}(\cdot)) \in L_{\oplus}^{1}(\Omega, \mathcal{F}, \mu ; \overline{\mathbb{R}})$. As $\bar{x}$ belongs to $X$, we conclude that $\bar{x}_{+} \in \underset{(1)}{\uparrow} X$, hence that $\underset{(1)}{\uparrow} X$ is not empty. Combining the three equations above, we readily get that

$$
\inf _{\mathfrak{u} \in \mathcal{U}} \int_{\Omega}^{*} g(\omega, \mathfrak{u}(\omega)) \mathrm{d} \mu(\omega)=\bigwedge_{x^{\prime} \in \in_{(1)}^{\uparrow}} \int_{\Omega} x^{\prime}(\omega) \mathrm{d} \mu(\omega) .
$$

- By [7, Proposition 5.4], as the function $g$ is a normal integrand and thus measurable, the set $\underset{(1)}{\uparrow} X$ is integrably inf-directed.

- The last step to obtain (18) is to prove that

$$
\inf _{u \in \mathbb{R}^{n}} g(\omega, u)={\underset{x^{\prime} \in \hat{\uparrow}}{(1)} X}_{\wedge} x^{\prime}(\omega)
$$

which is obtained using [7, Theorem 3.1].

\subsection{Comparison with Shapiro [14]}

A restricted literature $[12,14]$ considers interchange theorems not with integration but with more general monotone functionals. We focus on [14], which examines the case of three posets $\mathbb{X}$ : the set of continuous functions over a compact set; a Euclidean space; the normed linear space $L^{p}(\Omega, \mathcal{F}, \mu ; \mathbb{R})$ equipped with the $\mu$-pointwise order and its norm $\|\cdot\|_{p}$, where $p \in[1,+\infty]$ and $(\Omega, \mathcal{F}, \mu)$ is a probability space. For the sake simplicity, we will only consider the case $\mathbb{X}=L^{p}(\Omega, \mathcal{F}, \mu ; \overline{\mathbb{R}})$ and leave to the reader the two other cases, as they can be treated similarly.

We use the Minimization Interchange Theorem 2 to recover (an extended version of) the interchange result of [14].

Proposition 11. (extended from [14, Proposition 2.1]) Let $p \in[1,+\infty)$, $(\Omega, \mathcal{F}, \mu)$ be a probability space, $\left(L^{p}(\Omega, \mathcal{F}, \mu ; \mathbb{R}),\|\cdot\|_{p}\right)$ be the normed linear space of p-Lebesgue integrable functions equipped with the $\mu$-pointwise order, and $\Phi: L^{p}(\Omega, \mathcal{F}, \mu ; \mathbb{R}) \rightarrow \mathbb{R} \cup\{+\infty\}$ be an order preserving functional.

Let $\mathbb{U}$ be a set and $g: \Omega \times \mathbb{U} \rightarrow \overline{\mathbb{R}}$ be a function. We define the mapping $G: \mathbb{U}^{\Omega} \rightarrow \overline{\mathbb{R}}^{\Omega}$ by $G(\mathfrak{u}): \omega \ni \Omega \mapsto g(\omega, \mathfrak{u}(\omega)) \in \overline{\mathbb{R}}$, for all $\mathfrak{u} \in \mathbb{U}^{\Omega}$. We denote by $G^{b} \in \overline{\mathbb{R}}^{\Omega}$ the function $G^{b}: \omega \mapsto \inf _{u \in \mathbb{U}} g(\omega, u)$ and we assume that $G^{b} \in L^{p}(\Omega, \mathcal{F}, \mu ; \mathbb{R})$. Let $\mathcal{U} \subset \mathbb{U}^{\Omega}$ be a subset of $\mathbb{U}^{\Omega}$.

Suppose that 
$\left(S_{1}\right)$ the image $X=G(\mathcal{U})$ of $\mathcal{U} \subset \mathbb{U}^{\Omega}$ by the mapping $G: \mathbb{U}^{\Omega} \rightarrow \overline{\mathbb{R}}^{\Omega}$ is a subset of $L^{p}(\Omega, \mathcal{F}, \mu ; \mathbb{R})$, that is, $X=G(\mathcal{U}) \subset L^{p}(\Omega, \mathcal{F}, \mu ; \mathbb{R})$,

$\left(S_{2}\right)$ there exists a sequence $\left\{\mathfrak{u}_{n}\right\}_{n \in \mathbb{N}}$ in $\mathcal{U}$ such that

(a) $\left\|G\left(\mathfrak{u}_{n}\right)-G^{b}\right\|_{p} \underset{n \rightarrow+\infty}{\longrightarrow} 0$, that is, the sequence $\left\{G\left(\mathfrak{u}_{n}\right)\right\}_{n \in \mathbb{N}}$ (strongly) converges to $G^{b}$ in $\left(L^{p}(\Omega, \mathcal{F}, \mu ; \mathbb{R}),\|\cdot\|_{p}\right)$,

(b) $\Phi\left(G^{b}\right) \geq \underline{\lim }_{n \rightarrow+\infty} \Phi\left(G\left(\mathfrak{u}_{n}\right)\right)$ (an assumption which holds true when the mapping $\Phi: L^{p}(\Omega, \mathcal{F}, \mu ; \mathbb{R}) \rightarrow \mathbb{R} \cup\{+\infty\}$ is (strongly) continuous at $G^{b}$ ).

Then, we have that

$$
\inf _{x \in X} \Phi(x)=\Phi\left(G^{b}\right) .
$$

Proof. With the notation of Sect. 2 , we set $\mathbb{X}=L^{p}(\Omega, \mathcal{F}, \mu ; \mathbb{R})$ which is both a normed linear space and a poset when equipped with the $\mu$-pointwise order.

We prove Equation (19) by showing two equalities

$$
\bigwedge_{x \in X} x=G^{b} \text { and } \inf _{x \in X} \Phi(x)=\Phi\left(\bigwedge_{x \in X} x\right)
$$

where the right hand side is an interchange formula and the left hand side shows that the essential infimum over $X$ is realized by the pointwise infimum $G^{b}$, as done in [7, Theorem 3.1].

We prove the left hand side equality in Equation (20) as follows. First, we prove that the function $G^{b}$ satisfies $G^{b} \leq \inf _{\mathfrak{u} \in \mathcal{U}} G(\mathfrak{u})$. By definition of $G^{b}: \omega \mapsto \inf _{u \in \mathbb{U}} g(\omega, u)$, we have that $G^{b}(\omega) \leq G(\mathfrak{u})(\omega)=g(\omega, \mathfrak{u}(\omega))$ for all $\mathfrak{u} \in \mathcal{U}$ and $\omega \in \Omega$. Thus, we get that $G^{b} \leq G(\mathfrak{u})$ for all $\mathfrak{u} \in \mathcal{U}$.

Second, we prove that $G^{b}=\bigwedge_{\mathfrak{u} \in \mathcal{U}} G(\mathfrak{u})=\bigwedge_{n \in \mathbb{N}} G\left(\mathfrak{u}_{n}\right)$, where the sequence $\left\{\mathfrak{u}_{n}\right\}_{n \in \mathbb{N}}$ is given by Assumption $\left(S_{2} \mathrm{a}\right)$. Using the just proven property that $G^{b} \leq G(\mathfrak{u})$ for all $\mathfrak{u} \in \mathcal{U}$, and the fact that $\mathfrak{u}_{n} \in \mathcal{U}$ for all $n \in \mathbb{N}$, we obtain the following inequalities between functions in $\overline{\mathbb{R}}^{\Omega}$ :

$$
G^{b} \leq \bigwedge_{\mathfrak{u} \in \mathcal{U}} G(\mathfrak{u}) \leq \bigwedge_{n \in \mathbb{N}} G\left(\mathfrak{u}_{n}\right) \leq G\left(\mathfrak{u}_{0}\right)
$$

We now show that $G^{b}=\bigwedge_{n \in \mathbb{N}} G\left(\mathfrak{u}_{n}\right)$ where this equality is to be understood as an equality between classes in the space $L^{p}(\Omega, \mathcal{F}, \mu ; \mathbb{R})$. As $G(\mathcal{U}) \subset \mathbb{X}=$ $L^{p}(\Omega, \mathcal{F}, \mu ; \mathbb{R})$ by Assumption $\left(S_{1}\right)$, we get that $G\left(\mathfrak{u}_{0}\right) \in L^{p}(\Omega, \mathcal{F}, \mu ; \mathbb{R})$, hence so is $\wedge_{n \in \mathbb{N}} G\left(\mathfrak{u}_{n}\right)$. Now, as $0 \leq \wedge_{n^{\prime} \in \mathbb{N}} G\left(\mathfrak{u}_{n^{\prime}}\right)-G^{b} \leq G\left(\mathfrak{u}_{n}\right)-G^{b}$ for all $n \in \mathbb{N}$, 
we obtain the inequality $\left\|G^{b}-\bigwedge_{n^{\prime} \in \mathbb{N}} G\left(\mathfrak{u}_{n^{\prime}}\right)\right\|_{p} \leq\left\|G^{b}-G\left(\mathfrak{u}_{n}\right)\right\|_{p}$ between $L^{p}$-norms. As, by Assumption $\left(S_{2}\right.$ a), the sequence $\left\{G\left(\mathfrak{u}_{n}\right)\right\}_{n \in \mathbb{N}}$ strongly converges to $G^{b}$, we deduce that $G^{b}=\bigwedge_{n \in \mathbb{N}} G\left(\mathfrak{u}_{n}\right)$ in $L^{p}(\Omega, \mathcal{F}, \mu ; \mathbb{R})$. By $(21)$, we conclude that $G^{b}=\bigwedge_{\mathfrak{u} \in \mathcal{U}} G(\mathfrak{u})=\bigwedge_{n \in \mathbb{N}} G\left(\mathfrak{u}_{n}\right)$ in $L^{p}(\Omega, \mathcal{F}, \mu ; \mathbb{R})$.

Third, we define $x_{n}=G\left(\mathfrak{u}_{n}\right)$ for all $n \in \mathbb{N}$, with the property that the sequence $\left\{x_{n}\right\}_{n \in \mathbb{N}}$ is in $\mathbb{X}$ by Assumption $\left(S_{1}\right)$. Setting $\underline{x}=G^{b}$, we have obtained the following equalities in the poset $\mathbb{X}=L^{p}(\Omega, \mathcal{F}, \mu ; \mathbb{R})$ :

$$
\underline{x}=G^{b}=\bigwedge_{\mathfrak{u} \in \mathcal{U}} G(\mathfrak{u})=\bigwedge_{x \in X} x=\bigwedge_{n \in \mathbb{N}} x_{n} .
$$

We have thus proved the left hand side equality in Equation (20).

Now, we prove that the interchange formula in the right hand side of Equation (20) is satisfied by checking that the assumptions of Theorem 2 hold true. For this purpose, we define the subset $\widetilde{\mathbb{X}}=\uparrow\{\underline{x}\}=\{x \in \mathbb{X} \mid x \geq \underline{x}\}$ of $\mathbb{X}$. Then as a finite infimum of functions in $L^{p}(\Omega, \mathcal{F}, \mu ; \mathbb{R})$ is also in $L^{p}(\Omega, \mathcal{F}, \mu ; \mathbb{R})$, the subset $\widetilde{\mathbb{X}}$ is an inf-semilattice of the poset $\mathbb{X}$.

As the mapping $\Phi$ is order preserving, Assumption $\left(T_{1}\right)$ in Theorem 2 holds true. Also, we have already proven in (22) that Equation (6) holds true, which represents half of Assumption $\left(T_{2}\right)$ of Theorem 2. To prove (7), the second half of Assumption $\left(T_{2}\right)$ of Theorem 2, we consider the nondecreasing sequence $\left\{x_{n}^{\prime}\right\}_{n \in \mathbb{N}}$ in the inf-semilattice $\widetilde{\mathbb{X}}$ defined, for all $n \in \mathbb{N}$, by $x_{n}^{\prime}=$ $\wedge_{k \leq n} x_{k}$. As $\underline{x} \leq x_{n}^{\prime} \leq x_{n}$ for all $n \in \mathbb{N}$, and as the sequence $\left\{x_{n}\right\}_{n \in \mathbb{N}}$ strongly converges to $\underline{x}$, we readily get that so does the sequence $\left\{x_{n}^{\prime}\right\}_{n \in \mathbb{N}}$.

Now, we get that

$$
\begin{array}{rlr}
\Phi(\underline{x})=\Phi\left(G^{b}\right) & \geq \underline{\lim }_{n \rightarrow+\infty} \Phi\left(G\left(\mathfrak{u}_{n}\right)\right) & \text { (by Assumption } \left.\left(S_{2} \mathrm{~b}\right)\right) \\
& =\underline{\lim }_{n \rightarrow+\infty} \Phi\left(x_{n}\right) & \left(\text { as } x_{n}=G\left(\mathfrak{u}_{n}\right)\right) \\
& \geq \underline{\lim }_{n \rightarrow+\infty} \Phi\left(x_{n}^{\prime}\right) &
\end{array}
$$

as the mapping $\Phi$ is order preserving and as $x_{n} \geq x_{n}^{\prime}$

$$
=\bigwedge_{n \in \mathbb{N}} \Phi\left(x_{n}^{\prime}\right)
$$

as the sequence $\left\{x_{n}^{\prime}\right\}_{n \in \mathbb{N}}$ is nondecreasing, hence so is the sequence $\left\{\Phi\left(x_{n}^{\prime}\right)\right\}_{n \in \mathbb{N}}$. Thus, we have shown that (7) holds true.

As a consequence, we have shown that Assumption $\left(T_{2}\right)$ in Theorem 2 holds true. 
Finally, we prove that the subset $X$ is $\Phi$-inf-directed. For this purpose, we consider a finite subset $X^{\prime}$ of $X$. Recall that $\left\{x_{n}\right\}_{n \in \mathbb{N}}$ denotes a sequence in $X$ which strongly converges to $\underline{x}=\bigwedge_{x \in X} x$ and which realizes the infimum over $X$, i.e. $\wedge_{n \in \mathbb{N}} x_{n}=\bigwedge_{x \in X} x$. We successively have

$$
\begin{array}{rlr}
\Phi\left(\bigwedge_{x \in X^{\prime}} x\right) & \geq \Phi\left(\bigwedge_{x \in X} x\right) & \left(\text { as } \Phi \text { is order preserving and } \bigwedge_{x \in X^{\prime}} x \geq \wedge_{x \in X} x\right) \\
& =\Phi(\underline{x}), & \\
& \left.\geq \underline{\lim }_{n \rightarrow+\infty} \Phi\left(x_{n}\right) \quad \text { (by Assumption }\left(S_{2} \mathrm{~b}\right) \text { as } x_{n}=G\left(\mathfrak{u}_{n}\right)\right) \\
& \geq \bigwedge_{n \in \mathbb{N}} \Phi\left(x_{n}\right) \\
& \geq \bigwedge_{x \in X} \Phi(x), & \left(\text { as } x_{n} \in X \text { for all } n \in \mathbb{N}\right)
\end{array}
$$

which shows that $X$ is $\Phi$-inf-directed and ends the proof.

\subsection{Interchange between minimization and Choquet's integral}

Let $(\Omega, \mathcal{F})$ be a measurable space. We specialize the Minimization Interchange Theorem 2 to the poset of nonnegative measurable functions

$$
\mathbb{X}=\{x: \Omega \rightarrow \overline{\mathbb{R}} \mid x \geq 0 \text { and measurable }\}
$$

with the pointwise order and the Choquet integral $\Phi=\int^{\mathcal{C}}$ that we define below. We suggest [10] and the references therein for properties of the Choquet integral. One main difference of the Choquet integral compared to the Lebesgue integral is that it is nonadditive.

A capacity $c: \mathcal{F} \rightarrow \overline{\mathbb{R}}$ is a function which is order preserving $\left(\forall F_{1}, F_{2} \in\right.$ $\left.\mathcal{F}, F_{1} \subset F_{2} \Rightarrow c\left(F_{1}\right) \leq c\left(F_{2}\right)\right)$ and such that $c(\emptyset)=0$. Given a capacity $c$, the Choquet integral of a nonnegative measurable function $x \in \mathbb{X}$ is defined by

$$
\int_{\Omega}^{\mathcal{C}} x(\omega) \mathrm{d} c(\omega)=\int_{\mathbb{R}_{+}} c(x>t) \mathrm{d} t,
$$

where the integral on the right-hand side is the Lebesgue integral of an nonincreasing function. A capacity $c$ is said to be continuous from above if, for any nondecreasing sequence $\left\{F_{n}\right\}_{n \in \mathbb{N}} \subset \mathcal{F}$ of sets such that $F=\cap_{n \in \mathbb{N}} F_{n} \in \mathcal{F}$, 
we have that $c\left(F_{n}\right) \underset{n \rightarrow+\infty}{\longrightarrow} F$. Lastly, we say that a subset $X \subset \mathbb{X}$ of functions is Choquet integrably inf-directed if it is integrably inf-directed with the Choquet integral (23), as in Definition 1.

We readily get the following result, as an application of Theorem 2 .

Proposition 12. Let $(\Omega, \mathcal{F})$ be a measurable space, $\mathbb{X}=\{x: \Omega \rightarrow \overline{\mathbb{R}} \mid x \geq 0$ and measurable $\}$ be the poset of nonnegative measurable functions, and $c$ be a continuous from above capacity .

If $X=\left\{x_{i}\right\}_{i \in I} \subset \mathbb{X}$ is a family of nonnegative measurable functions with the countable inf property, we have that

$$
\bigwedge_{i \in I} \int_{\Omega}^{\mathcal{C}} x_{i} \mathrm{~d} c=\int_{\Omega}^{\mathcal{C}} \bigwedge_{i \in I} x_{i} \mathrm{~d} c
$$

if, and only if, $X$ is Choquet integrably inf-directed.

Proof. We check that the assumptions of Theorem 2 are satisfied.

- The set $\mathbb{X}=\{x: \Omega \rightarrow \overline{\mathbb{R}} \mid x \geq 0$ and measurable $\}$ of nonnegative measurable functions endowed with the pointwise order is an inf-semilattice.

- The Choquet integral is order preserving on $\mathbb{X}$ (see [10, Proposition 2.3]).

- As the capacity $c$ is countinuous from above, the following monotone pointwise convergence theorem holds (see [10, Theorem 3.2.(2)]): for every nonincreasing sequence $\left\{x_{n}\right\}_{n \in \mathbb{N}}$ of functions converging pointwise to $x \in \mathbb{X}$, we have that

$$
\bigwedge_{n \in \mathbb{N}} \int_{\Omega}^{\mathcal{C}} x_{n} \mathrm{~d} c=\lim _{n \in \mathbb{N}} \int_{\Omega}^{\mathcal{C}} x_{n} \mathrm{~d} c=\int_{\Omega}^{\mathcal{C}} x \mathrm{~d} c .
$$

As a consequence, the Choquet integral is sequentially-inf-continuous on $\mathbb{X}$ (see Definition 3).

Hence, by Theorem 2, given $X=\left\{x_{i}\right\}_{i \in I} \subset \mathbb{X}$ a family of nonnegative functions with the countable inf property, we have

$$
\bigwedge_{i \in I} \int_{\Omega}^{\mathcal{C}} x_{i} \mathrm{~d} c=\int_{\Omega}^{\mathcal{C}} \bigwedge_{i \in I} x_{i} \mathrm{~d} c
$$

if, and only if, the subset $X$ is Choquet integrably inf-directed. 
One could get a similar interchange result between Choquet integral and maximization on subsets of nonpositive measurable functions by setting, for every nonpositive measurable function $x, \int_{\Omega}^{\mathcal{C}} x \mathrm{~d} c=-\int_{\Omega}^{\mathcal{C}}(-x) \mathrm{d} c$, where the right hand side is the Choquet integral (23) for nonnegative measurable functions.

\section{Conclusion}

As recalled in the introduction, the question of interchanging integration and minimization is an important issue in optimization (especially in stochastic optimization where integration corresponds to mathematical expectation or to risk measure). By using the framework of posets - and especially the notions of semilattice, $\Phi$-inf-directed subset, inf countable subset - we have provided an umbrella theorem that covers a wide spectrum of results (and extends them). Moreover, our approach goes beyond integration and is able to handle more general monotone functionals.

\section{A Extended Lebesgue and outer integrals}

The set $\overline{\mathbb{R}}=\mathbb{R} \cup\{+\infty\} \cup\{-\infty\}$ is endowed with its Borel $\sigma$-algebra (see [11, Chap. II]), and with the following extended additions and multiplication. We still denote by + the usual addition when extended to $\overline{\mathbb{R}}_{+}=\mathbb{R} \cup\{+\infty\}$ by $+\infty$ being absorbant, and to $\overline{\mathbb{R}}_{-}=\mathbb{R} \cup\{-\infty\}$ by $-\infty$ being absorbant. Then, we denote by + the addition on $\overline{\mathbb{R}}$ for which $-\infty$ is absorbant, i.e. $(+\infty)+(-\infty)=(-\infty)+(+\infty)=-\infty$ and by $\dot{+}$ the addition for which $+\infty$ is absorbant, i.e. $(+\infty) \dot{+}(-\infty)=(-\infty) \dot{+}(+\infty)=+\infty$. We set $\lambda \times( \pm \infty)= \pm \infty$ for $\lambda \in] 0,+\infty[, \lambda \times( \pm \infty)=\mp \infty$ for $\lambda \in]-\infty, 0[$, and $0 \times( \pm \infty)=0$.

Throughout this section, we fix a $\sigma$-finite measured space $(\Omega, \mathcal{F}, \mu)$. The classical Lebesgue integral w.r.t. the $\sigma$-finite measure $\mu$ is defined for functions with values in $\mathbb{R}$ (real-valued functions). As we are motivated by optimization, we need results for integrals of functions with values in $\overline{\mathbb{R}}$ (extended real-valued functions). For integration of measurable real-valued functions w.r.t. a $\sigma$-finite measure $\mu$, we refer the reader to [2, Chapter 11]; for integration of measurable extended real-valued functions w.r.t. a probability measure $\mu$, we refer the reader to [11]; for integration of measurable extended 
real-valued functions w.r.t. a $\sigma$-finite measure $\mu$, we refer the reader to $[8$, Chapter V]; for outer integration of extended real-valued functions w.r.t. a $\sigma$-finite measure $\mu$, we refer the reader to [3].

It happens that results about monotonicity, additivity, external multiplication and monotone convergence of the integral are either scattered in the literature, or sometimes not formulated. This is due to the fact that the extension of the Lebesgue integral to extended real-valued functions gives rise to different expressions, which renders the exposition less systematic and elegant than with the Lebesgue integral of integrable real-valued functions. Also, some results belong to folklore and its is hard to find trace of their proof, as they are considered obvious. However, for the purpose of optimizing integral expressions, we provide below a systematic exposition of the functional spaces $L^{0}(\Omega, \mathcal{F}, \mu ; \overline{\mathbb{R}}), L_{\oplus}^{1}(\Omega, \mathcal{F}, \mu ; \overline{\mathbb{R}})$ and $L_{\ominus}^{1}(\Omega, \mathcal{F}, \mu ; \overline{\mathbb{R}})$, and how the Lebesgue integral can be extended.

\section{A.1 Functional space $L^{0}(\Omega, \mathcal{F}, \mu ; \overline{\mathbb{R}})$ and the Lebesgue integral}

We endow the set $\overline{\mathbb{R}}^{\Omega}$ of functions $f: \Omega \rightarrow \overline{\mathbb{R}}$ with the $\mu$-pointwise order $\leq$ as follows: for any $f, g \in \overline{\mathbb{R}}^{\Omega}$,

$$
f \leq g \Longleftrightarrow \exists A \in \mathcal{F}, \quad \mu(A)=0, \quad f(\omega) \leq g(\omega), \forall \omega \in \Omega \backslash A
$$

We denote by $\mathcal{L}^{0}(\Omega, \mathcal{F} ; \overline{\mathbb{R}})$ the set of measurable functions from $\Omega$ to $\overline{\mathbb{R}}$ and by $L^{0}(\Omega, \mathcal{F}, \mu ; \overline{\mathbb{R}})$ the quotient $\mathcal{L}^{0}(\Omega, \mathcal{F} ; \overline{\mathbb{R}}) / \sim$ where for any $f, g \in$ $\mathcal{L}^{0}(\Omega, \mathcal{F} ; \overline{\mathbb{R}}), f \sim g$ if, and only if, $f=g \mu$-almost everywhere. The $\mu$ pointwise order (24) induces an order on the set $L^{0}(\Omega, \mathcal{F}, \mu ; \overline{\mathbb{R}})$ of equivalence classes, that we will also denote by $\leq$ and call the $\mu$-pointwise order. Thus, the expression $f \geq 0$ makes sense for $f \in L^{0}(\Omega, \mathcal{F}, \mu ; \overline{\mathbb{R}})$. In the same way, we introduce the $\mu$-pointwise strict order $<$ on the set $L^{0}(\Omega, \mathcal{F}, \mu ; \overline{\mathbb{R}})$ of equivalence classes: $f<g \Longleftrightarrow f \leq g$ and $f \neq g$. Thus, the expressions $-\infty<f$, $f<+\infty$ and $-\infty<f<+\infty$ make sense for $f \in L^{0}(\Omega, \mathcal{F}, \mu ; \overline{\mathbb{R}})$.

The set $L^{0}(\Omega, \mathcal{F}, \mu ; \overline{\mathbb{R}})$ is stable under the two additions + or $\dot{+}$, and under external multiplication. We say that a subset of $L^{0}(\Omega, \mathcal{F}, \mu ; \overline{\mathbb{R}})$ is a convex cone, if it is stable under the addition + and under external multiplication by a scalar in $\mathbb{R}_{+}$.

We write $\int$ for the Lebesgue integral deduced from the $\sigma$-finite measured 
space $(\Omega, \mathcal{F}, \mu)$. The Lebesgue integral $\int$ is defined on the convex cone

$$
L_{+}^{0}(\Omega, \mathcal{F}, \mu ; \overline{\mathbb{R}})=\left\{f \in L^{0}(\Omega, \mathcal{F}, \mu ; \overline{\mathbb{R}}) \mid f \geq 0\right\},
$$

where it takes values in $\overline{\mathbb{R}}_{+}$, given by the formula (see [2, Footnote 3 , p. 411] for real-valued functions)

$$
\int f=\int f \mathrm{~d} \mu=\sup \left\{\int_{\Omega} \varphi \mathrm{d} \mu \mid 0 \leq \varphi \leq f, \varphi \text { simple and nonnegative }\right\},
$$

where simple nonnegative functions (or $\mu$-step functions) are functions of the form $\varphi(\cdot)=\sum_{i \in I} \alpha_{i} \mathbf{1}_{A_{i}}(\cdot)$ with $I$ finite and $\left\{A_{i}\right\}_{i \in I}$ a sequence of measurable sets such that $\mu\left(A_{i}\right)<+\infty$ for all $i \in I$ and the coefficients $\left\{\alpha_{i}\right\}_{i \in I}$ are nonnegative and finite reals and the indicator function $\mathbf{1}_{A}$ of a subset of $\Omega$ is defined by $\mathbf{1}_{A}(x)=1$ if $x \in A$ and $\mathbf{1}_{A}(x)=0$ if $x \notin A$.

The (extended) Lebesgue integral on $L_{+}^{0}(\Omega, \mathcal{F}, \mu ; \overline{\mathbb{R}})$ satisfies the following properties

- monotone: $\forall f, g \in L_{+}^{0}(\Omega, \mathcal{F}, \mu ; \overline{\mathbb{R}}), f \leq g \Longrightarrow \int f \leq \int g$,

- additive: $\forall f, g \in L_{+}^{0}(\Omega, \mathcal{F}, \mu ; \overline{\mathbb{R}}), \int(f+g)=\int f+\int g$,

- positively homogeneous: $\forall f \in L_{+}^{0}(\Omega, \mathcal{F}, \mu ; \overline{\mathbb{R}}), \forall \lambda \in \mathbb{R}_{+}, \int(\lambda f)=\lambda \int f$,

- monotone convergence: for any nondecreasing sequence $\left(f_{n}\right)_{n \in \mathbb{N}}$ in $L_{+}^{0}(\Omega, \mathcal{F}, \mu ; \overline{\mathbb{R}})$, then $f=\sup _{n \in \mathbb{N}} f_{n} \in L_{+}^{0}(\Omega, \mathcal{F}, \mu ; \overline{\mathbb{R}})$ and $\lim _{n \rightarrow+\infty} \int f_{n}=\int f$.

\section{A.2 Functional spaces $L_{\oplus}^{1}(\Omega, \mathcal{F}, \mu ; \overline{\mathbb{R}}), L_{\ominus}^{1}(\Omega, \mathcal{F}, \mu ; \overline{\mathbb{R}})$ and the extended Lebesgue integral}

For any function $f: \Omega \rightarrow \overline{\mathbb{R}}$, we define its positive part $f_{+}=\sup (0, f)$ and its negative part $f_{-}=\sup (0,-f)$. Obviously, we have $f=f_{+}+\left(-f_{-}\right)$(where we use the addition + as one of the terms is zero for any value taken by the argument of the function $f$ ). We define the set

$$
\mathcal{L}_{\oplus}^{1}(\Omega, \mathcal{F} ; \overline{\mathbb{R}})=\left\{f \in \mathcal{L}^{0}(\Omega, \mathcal{F} ; \overline{\mathbb{R}}) \mid \int_{\Omega} f_{+} \mathrm{d} \mu<+\infty\right\}
$$

and the quotient set $\mathcal{L}_{\oplus}^{1}(\Omega ; \overline{\mathbb{R}}) \backslash \sim$ by

$$
L_{\oplus}^{1}(\Omega, \mathcal{F}, \mu ; \overline{\mathbb{R}})=\left\{f \in L^{0}(\Omega, \mathcal{F}, \mu ; \overline{\mathbb{R}}) \mid \int_{\Omega} f_{+} \mathrm{d} \mu<+\infty\right\},
$$


with the property that

$$
f \in L_{\oplus}^{1}(\Omega, \mathcal{F}, \mu ; \overline{\mathbb{R}}) \Longrightarrow f<+\infty
$$

because $\int_{\Omega} f_{+} \mathrm{d} \mu<+\infty \Longrightarrow f_{+}<+\infty \Longrightarrow f \leq f_{+}<+\infty$. In the same way, we define

$$
\begin{aligned}
\mathcal{L}_{\ominus}^{1}(\Omega, \mathcal{F} ; \overline{\mathbb{R}}) & =\left\{f \in \mathcal{L}^{0}(\Omega, \mathcal{F} ; \overline{\mathbb{R}}) \mid \int_{\Omega} f_{-} \mathrm{d} \mu<+\infty\right\}, \\
L_{\ominus}^{1}(\Omega, \mathcal{F}, \mu ; \overline{\mathbb{R}}) & =\left\{f \in L^{0}(\Omega, \mathcal{F}, \mu ; \overline{\mathbb{R}}) \mid \int_{\Omega} f_{-} \mathrm{d} \mu<+\infty\right\},
\end{aligned}
$$

with the properties that $L_{\ominus}^{1}(\Omega, \mathcal{F}, \mu ; \overline{\mathbb{R}})=-L_{\oplus}^{1}(\Omega, \mathcal{F}, \mu ; \overline{\mathbb{R}})$ and that $f \in$ $L_{\ominus}^{1}(\Omega, \mathcal{F}, \mu ; \overline{\mathbb{R}}) \Longrightarrow-\infty<f$.

We say that a (class of) function $(\mathrm{s}) f \in L^{0}(\Omega, \mathcal{F}, \mu ; \overline{\mathbb{R}})$ is semi-integrable if it belongs to $L_{\oplus}^{1}(\Omega, \mathcal{F}, \mu ; \overline{\mathbb{R}}) \cup L_{\ominus}^{1}(\Omega, \mathcal{F}, \mu ; \overline{\mathbb{R}})$, that is, if either $\int_{\Omega} f_{+} \mathrm{d} \mu<+\infty$ or $\int_{\Omega} f_{-} \mathrm{d} \mu<+\infty$. The Lebesgue integral is extended from the convex cone $L_{+}^{0}(\Omega, \mathcal{F}, \mu ; \overline{\mathbb{R}})$ to semi-integrable functions by ([11, Proposition II-3-2], $[2$, Chapter 11], [8, Chapter V])

$$
\int f=\int f_{+}+\left(-\int f_{-}\right), \forall f \in L_{\oplus}^{1}(\Omega, \mathcal{F}, \mu ; \overline{\mathbb{R}}) \cup L_{\ominus}^{1}(\Omega, \mathcal{F}, \mu ; \overline{\mathbb{R}}),
$$

where we use the addition + as one of the terms is zero. The extended Lebesgue integral on semi-integrable functions satisfies the following properties (listed in [11, Proposition II-3-3])

- monotone: $\forall f, g \in L_{\oplus}^{1}(\Omega, \mathcal{F}, \mu ; \overline{\mathbb{R}}) \cup L_{\ominus}^{1}(\Omega, \mathcal{F}, \mu ; \overline{\mathbb{R}}), f \leq g \Longrightarrow \int f \leq$ $\int g$

- additive on $L_{\oplus}^{1}(\Omega, \mathcal{F}, \mu ; \overline{\mathbb{R}}): \forall f, g \in L_{\oplus}^{1}(\Omega, \mathcal{F}, \mu ; \overline{\mathbb{R}}), \int(f+g)=\int f+$ $\int g$

- additive on $L_{\ominus}^{1}(\Omega, \mathcal{F}, \mu ; \overline{\mathbb{R}}): \forall f, g \in L_{\ominus}^{1}(\Omega, \mathcal{F}, \mu ; \overline{\mathbb{R}}), \int(f+g)=\int f+$ $\int g$

- positively and negatively homogeneous: $\forall f \in L_{\oplus}^{1}(\Omega, \mathcal{F}, \mu ; \overline{\mathbb{R}}) \cup L_{\ominus}^{1}(\Omega, \mathcal{F}, \mu ; \overline{\mathbb{R}})$, $\forall \lambda \in \mathbb{R}, \int(\lambda f)=\lambda \int f$

- monotone convergence on $L_{\oplus}^{1}(\Omega, \mathcal{F}, \mu ; \overline{\mathbb{R}})$ : for any nonincreasing sequence $\left(f_{n}\right)_{n \in \mathbb{N}}$ in $L_{\oplus}^{1}(\Omega, \mathcal{F}, \mu ; \overline{\mathbb{R}})$, then $f=\inf _{n \in \mathbb{N}} f_{n} \in L_{\oplus}^{1}(\Omega, \mathcal{F}, \mu ; \overline{\mathbb{R}})$ and $f_{n} \downarrow f$ and $\lim _{n \rightarrow+\infty} \int f_{n}=\int f$, 
- monotone convergence on $L_{\ominus}^{1}(\Omega, \mathcal{F}, \mu ; \overline{\mathbb{R}})$ : for any nondecreasing sequence $\left(f_{n}\right)_{n \in \mathbb{N}}$ in $L_{\ominus}^{1}(\Omega, \mathcal{F}, \mu ; \overline{\mathbb{R}})$, then $f=\sup _{n \in \mathbb{N}} f_{n} \in L_{\ominus}^{1}(\Omega, \mathcal{F}, \mu ; \overline{\mathbb{R}})$ and $f_{n} \uparrow f$ and $\lim _{n \rightarrow+\infty} \int f_{n}=\int f$.

We provide some of the proofs.

Lemma 13. For any functions $f$ and $g$ in $L_{\ominus}^{1}(\Omega, \mathcal{F}, \mu ; \overline{\mathbb{R}})$, we have $f+g \in$ $L_{\ominus}^{1}(\Omega, \mathcal{F}, \mu ; \overline{\mathbb{R}})$ and

$$
\int_{\Omega}(f+g) \mathrm{d} \mu=\int_{\Omega} f \mathrm{~d} \mu+\int_{\Omega} g \mathrm{~d} \mu, \quad \forall f \in L_{\ominus}^{1}(\Omega, \mathcal{F}, \mu ; \overline{\mathbb{R}}), g \in L_{\ominus}^{1}(\Omega, \mathcal{F}, \mu ; \overline{\mathbb{R}}) .
$$

Proof. We consider $f, g \in L_{\ominus}^{1}(\Omega, \mathcal{F}, \mu ; \overline{\mathbb{R}})$. Notice that, as $f, g \in L_{\ominus}^{1}(\Omega, \mathcal{F}, \mu ; \overline{\mathbb{R}})$, we have that $-\infty<f$ and $-\infty<g$, so that we will use the addition + .

- We show that $\int_{\Omega}(f+g) \mathrm{d} \mu<+\infty$. On the one hand, we have

$$
(f+g)_{-}=\sup (0,-(f+g))=\sup (0,(-f)+(-g)) .
$$

On the other hand, we have $(-f) \leq f_{-}$and $(-g) \leq g_{-}$, hence $(-f)+(-g) \leq$ $f_{-}+g_{-}$and thus $(f+g)_{-} \leq f_{-}+g_{-}$. By monotonicity and additivity of the Lebesgue integral on $L_{+}^{0}(\Omega, \mathcal{F}, \mu ; \overline{\mathbb{R}})$, we deduce that

$$
\int_{\Omega}(f+g)_{-} \mathrm{d} \mu \leq \int_{\Omega} f_{-} \mathrm{d} \mu+\int_{\Omega} g_{-} \mathrm{d} \mu<+\infty
$$

because $\int_{\Omega} f_{-} \mathrm{d} \mu<+\infty$ and $\int_{\Omega} g_{-} \mathrm{d} \mu<+\infty$ by assumption $\left(f, g \in L_{\ominus}^{1}(\Omega, \mathcal{F}, \mu ; \overline{\mathbb{R}})\right)$. Hence, $f+g \in L_{\ominus}^{1}(\Omega, \mathcal{F}, \mu ; \mathbb{R})$.

- We prove the additivity of the integral. Notice that, as $f, g, f+g \in$ $L_{\ominus}^{1}(\Omega, \mathcal{F}, \mu ; \overline{\mathbb{R}})$, we have that $-\infty<f$ and $-\infty<g$, and also that $0 \leq$ $f_{-}<+\infty, 0 \leq g_{-}<+\infty, 0 \leq(f+g)_{-}<+\infty, 0 \leq \int_{\Omega} f_{-} \mathrm{d} \mu<+\infty, 0 \leq$ $\int_{\Omega} g_{-} \mathrm{d} \mu<+\infty, 0 \leq \int_{\Omega}(f+g)_{-}<+\infty$, so that we will use the addition + .

As, for any function $h$, we have that $h=h_{+}+\left(-h_{-}\right.$) (where we use the addition + as one of the terms is zero), we immediately get that

$$
(f+g)_{+}+\left(-(f+g)_{-}\right)=f+g=f_{+}+\left(-f_{-}\right)+g_{+}+\left(-g_{-}\right) .
$$

Now, if we add, to the left and right hand side of the above equality, the three nonnegative reals $(f+g)_{-}, f_{-}$and $g_{-}$(none of them being $\left.+\infty\right)$, we obtain the equality

$$
(f+g)_{+}+f_{-}+g_{-}=f_{+}+g_{+}+(f+g)_{-} .
$$


As this is an equality between sums of nonnegative functions, we apply the Lebesgue integral on $L_{+}^{0}(\Omega, \mathcal{F}, \mu ; \overline{\mathbb{R}})$, and get that

$\int_{\Omega}(f+g)_{+} \mathrm{d} \mu+\int_{\Omega} f_{-} \mathrm{d} \mu+\int_{\Omega} g_{-} \mathrm{d} \mu=\int_{\Omega} f_{+} \mathrm{d} \mu+\int_{\Omega} g_{+} \mathrm{d} \mu+\int_{\Omega}(f+g)_{-} \mathrm{d} \mu$,

by additivity of the Lebesgue integral on $L_{+}^{0}(\Omega, \mathcal{F}, \mu ; \overline{\mathbb{R}})$. Now, the quantities $\int_{\Omega} f_{-} \mathrm{d} \mu, \int_{\Omega} g_{-} \mathrm{d} \mu$ and $\int_{\Omega}(f+g)_{-} \mathrm{d} \mu$ are three nonnegative reals (none of them being $+\infty)$ by assumption $\left(f, g \in L_{\ominus}^{1}(\Omega, \mathcal{F}, \mu ; \overline{\mathbb{R}})\right.$ and property $f+g \in$ $\left.L_{\ominus}^{1}(\Omega, \mathcal{F}, \mu ; \overline{\mathbb{R}})\right)$. Thus, we get, by subtracting these three finite terms,

$$
\begin{array}{r}
\int_{\Omega}(f+g)_{+} \mathrm{d} \mu+\left(-\int_{\Omega}(f+g)_{-} \mathrm{d} \mu\right) \\
=\int_{\Omega} f_{+} \mathrm{d} \mu+\left(-\int_{\Omega} f_{-} \mathrm{d} \mu\right)+\int_{\Omega} g_{+} \mathrm{d} \mu+\left(-\int_{\Omega} g_{-} \mathrm{d} \mu\right),
\end{array}
$$

hence, by (29),

$$
\int_{\Omega}(f+g) \mathrm{d} \mu=\int_{\Omega} f \mathrm{~d} \mu+\int_{\Omega} g \mathrm{~d} \mu .
$$

This ends the proof.

Lemma 14. We have

$$
\int_{\Omega}(-f) \mathrm{d} \mu=-\int_{\Omega} f \mathrm{~d} \mu, \quad \forall f \in L_{\oplus}^{1}(\Omega, \mathcal{F}, \mu ; \overline{\mathbb{R}}) \cup L_{\ominus}^{1}(\Omega, \mathcal{F}, \mu ; \overline{\mathbb{R}}) .
$$

Proof. This is an obvious consequence of $(29)$, and of $(-f)_{+}=f_{-}$and $(-f)_{-}=f_{+}$.

Proposition 15 (Extended monotone convergence theorem for $L_{\oplus}^{1}(\Omega, \mathcal{F}, \mu ; \overline{\mathbb{R}})$ ). Let $\left(f_{n}\right)_{n \in \mathbb{N}}$ be an nonincreasing sequence of functions in $L_{\oplus}^{1}(\Omega, \mathcal{F}, \mu ; \overline{\mathbb{R}})$, converging to $f \in \overline{\mathbb{R}}^{\Omega}$, that is, $f_{n} \downarrow f$. Then, $f \in L_{\oplus}^{1}(\Omega, \mathcal{F}, \mu ; \overline{\mathbb{R}})$ and we have that

$$
\lim _{n \rightarrow+\infty} \int f_{n} d \mu=\int f d \mu
$$

Proof. Let $\left(f_{n}\right)_{n \in \mathbb{N}}$ be an nonincreasing sequence of functions in $L_{\oplus}^{1}(\Omega, \mathcal{F}, \mu ; \overline{\mathbb{R}})$, such that $f_{n} \downarrow f$. As $f_{n} \in L_{\oplus}^{1}(\Omega, \mathcal{F}, \mu ; \overline{\mathbb{R}})$, we have that $f_{n}<+\infty$ for all $n \in \mathbb{N}$, so that we will use the addition + . 
As $f \leq f_{1}$, we have that $\sup (0, f)=f_{+} \leq\left(f_{1}\right)_{+}=\sup \left(0, f_{1}\right)$, hence $\int f_{+} d \mu \leq \int\left(f_{1}\right)_{+} d \mu<+\infty$, where the last strict inequality is by assumption $\left(f_{1} \in L_{\oplus}^{1}(\Omega, \mathcal{F}, \mu ; \overline{\mathbb{R}})\right)$. We conclude that $f \in L_{\oplus}^{1}(\Omega, \mathcal{F}, \mu ; \overline{\mathbb{R}})$.

As, by assumption, $\int\left(f_{1}\right)_{+} d \mu<+\infty$, we conclude that $\left(f_{1}\right)_{+}<+\infty$. We consider two cases.

We suppose that $\int\left(f_{1}\right)_{-} d \mu=+\infty$. As $\sup (0,-f)=f_{-} \geq\left(f_{1}\right)_{-}=$ $\sup \left(0,-f_{1}\right)$, we also have that $\int f_{-} d \mu=+\infty$. As a consequence, we get that $\int f_{-} d \mu=\int\left(f_{1}\right)_{-} d \mu=+\infty$, hence $\int f d \mu=\int f_{1} d \mu=+\infty$, by definition of the integral $\int$ on $L_{\oplus}^{1}(\Omega, \mathcal{F}, \mu ; \overline{\mathbb{R}})$. By monotonicity of the integral $\int$, we conclude that $+\infty=\int f_{1} d \mu \leq \lim _{n \rightarrow+\infty} \int f_{n} d \mu \leq \int f_{1} d \mu=+\infty$, hence that (30) holds true.

We now suppose that $\int\left(f_{1}\right)_{-} d \mu<+\infty$. We deduce that $\left(f_{1}\right)_{-}<+\infty$. As we had $\left(f_{1}\right)_{+}<+\infty$, we deduce that $-\infty<f_{1}<+\infty$. Thus, we can define $\varphi_{n}=f_{n}+\left(-f_{1}\right)$ and $\varphi=f+\left(-f_{1}\right)$, which are functions in $L^{0}(\Omega, \mathcal{F}, \mu ; \overline{\mathbb{R}})$ such that $\varphi=f+\left(-f_{1}\right) \leq \varphi_{n}=f_{n}+\left(-f_{1}\right) \leq 0$, because $f_{n} \leq f_{1}$. As $f_{1}$ takes values in $\mathbb{R}$, we have that $\inf _{n}\left(f_{n}+\left(-f_{1}\right)\right)=\inf _{n} f_{n}+\left(-f_{1}\right)$, hence we obtain that $\varphi_{n} \downarrow \varphi$. As $\varphi_{n} \leq 0$, by the monotone convergence theorem for $\left(L_{-}^{0}(\Omega, \mathcal{F}, \mu ; \overline{\mathbb{R}}), \int\right)$, we get that

$$
\inf _{n} \int \varphi_{n} d \mu=\lim _{n \rightarrow+\infty} \int \varphi_{n} d \mu=\int \varphi d \mu
$$

As, by assumption, $\int\left(f_{1}\right)_{-} d \mu<+\infty$ and $\int\left(f_{1}\right)_{+} d \mu<+\infty$, we get that $f_{1} \in L^{1}(\Omega, \mathcal{F}, \mu ; \overline{\mathbb{R}})$ and that $-\infty<\int f_{1} d \mu<+\infty$, hence obtaining

$$
\inf _{n}\left(\int \varphi_{n} d \mu+\int f_{1} d \mu\right)=\inf _{n} \int \varphi_{n} d \mu+\int f_{1} d \mu=\int \varphi d \mu+\int f_{1} d \mu .
$$

As $\varphi_{n} \leq 0$ and belongs to $L^{0}(\Omega, \mathcal{F}, \mu ; \overline{\mathbb{R}})$, we have that $\varphi_{n} \in L_{\oplus}^{1}(\Omega, \mathcal{F}, \mu ; \overline{\mathbb{R}})$. In the same way, we obtain that $\varphi \in L_{\oplus}^{1}(\Omega, \mathcal{F}, \mu ; \overline{\mathbb{R}})$. By the + -additivity property of the integral $\int$ on $L_{\oplus}^{1}(\Omega, \mathcal{F}, \mu ; \overline{\mathbb{R}})$, we calculate the first and last terms of the above equality, and we obtain

$$
\inf _{n} \int\left(\varphi_{n}+f_{1}\right) d \mu=\int\left(\varphi+f_{1}\right) d \mu
$$

We obtain (30) because $\varphi_{n}+f_{1}=f_{n}+\left(-f_{1}\right)+f_{1}=f_{n}$ since $f_{1}$ takes values in $\mathbb{R}$, and, in the same way, $\varphi+f_{1}=f+\left(-f_{1}\right)+f_{1}=f$. 
The classical vector space of integrable functions is

$$
L^{1}(\Omega, \mathcal{F}, \mu ; \overline{\mathbb{R}})=L_{\oplus}^{1}(\Omega, \mathcal{F}, \mu ; \overline{\mathbb{R}}) \cap L_{\ominus}^{1}(\Omega, \mathcal{F}, \mu ; \overline{\mathbb{R}})
$$

with the property that $f \in L^{1}(\Omega, \mathcal{F}, \mu ; \overline{\mathbb{R}}) \Longrightarrow-\infty<f<+\infty$, that is, $L^{1}(\Omega, \mathcal{F}, \mu ; \overline{\mathbb{R}})=L^{1}(\Omega, \mathcal{F}, \mu ; \mathbb{R})$.

\section{A.3 Outer integral on $L^{0}(\Omega, \mathcal{F}, \mu ; \overline{\mathbb{R}})$}

We follow [3] for the following definitions.

Definition 16. We define the outer integral of a function by

$$
\int_{\Omega}^{*} f \mathrm{~d} \mu=\inf \left\{\int_{\Omega} \psi \mathrm{d} \mu \mid \psi \in L^{1}(\Omega, \mathcal{F}, \mu ; \mathbb{R}) \text { and } f \leq \psi\right\}, \forall f \in \overline{\mathbb{R}}^{\Omega},
$$

and the inner integral by

$$
\int_{*}^{\Omega} f \mathrm{~d} \mu=\sup \left\{\int_{\Omega} \psi \mathrm{d} \mu \mid \psi \in L^{1}(\Omega, \mathcal{F}, \mu ; \mathbb{R}) \text { and } f \geq \psi\right\}, \forall f \in \overline{\mathbb{R}}^{\Omega}
$$

where $\int_{\Omega} \psi \mathrm{d} \mu$ is the classical Lebesgue integral for $\psi \in L^{1}(\Omega, \mathcal{F}, \mu ; \mathbb{R})$.

It is straightforward that

$$
\begin{gathered}
\int_{*}^{\Omega} f \mathrm{~d} \mu \leq \int_{\Omega}^{*} f \mathrm{~d} \mu, \forall f \in \overline{\mathbb{R}}^{\Omega}, \\
-\int_{\Omega}^{*} f \mathrm{~d} \mu \leq \int_{\Omega}^{*}(-f) d \mu, \quad \forall f \in \overline{\mathbb{R}}^{\Omega}, \\
\int_{*}^{\Omega} f \mathrm{~d} \mu=-\left(\int_{\Omega}^{*}(-f) \mathrm{d} \mu\right), \forall f \in \overline{\mathbb{R}}^{\Omega} .
\end{gathered}
$$

These outer and inner integrals extend the classical Lebesgue integral to the uncovered case where both $\int_{\Omega} f_{+} \mathrm{d} \mu$ and $\int_{\Omega} f_{-} \mathrm{d} \mu$ equal $+\infty$ as shown in the following Proposition. 
Proposition 17. We have that

$$
\begin{aligned}
& \int_{\Omega}^{*} f \mathrm{~d} \mu=\int_{\Omega} f_{+} \mathrm{d} \mu \dot{+}\left(-\int_{\Omega} f_{-} \mathrm{d} \mu\right), \quad \forall f \in L^{0}(\Omega, \mathcal{F}, \mu ; \overline{\mathbb{R}}), \\
& \int_{*}^{\Omega} f \mathrm{~d} \mu=\int_{\Omega} f_{+} \mathrm{d} \mu+\left(-\int_{\Omega} f_{-} \mathrm{d} \mu\right), \quad \forall f \in L^{0}(\Omega, \mathcal{F}, \mu ; \overline{\mathbb{R}}) .
\end{aligned}
$$

As a consequence, the outer integral of $f$ coincides with the extended Lebesgue integral $(29)$ on $L_{\oplus}^{1}(\Omega, \mathcal{F}, \mu ; \overline{\mathbb{R}}) \cup L_{\ominus}^{1}(\Omega, \mathcal{F}, \mu ; \overline{\mathbb{R}})$, that is, when $f$ is semiintegrable.

Proof. We consider $f \in L^{0}(\Omega, \mathcal{F}, \mu ; \overline{\mathbb{R}})$ and we examine four possible cases in order to prove Equation (34a) (then Equation (34b) is obtained from (33c)).

- Suppose that $\int_{\Omega} f_{+} \mathrm{d} \mu<+\infty$ and $\int_{\Omega} f_{-} \mathrm{d} \mu<+\infty$ (that is, $\left.f \in L^{1}(\Omega, \mathcal{F}, \mu ; \mathbb{R})\right)$. Then we have that $\mu[\{f= \pm \infty\}]=0$, and thus there exists a representant $\tilde{f} \in L^{1}(\Omega, \mathcal{F}, \mu ; \mathbb{R})$ in the class, which is equal to $f(\mu$-a.s. $)$. Thus, we have that $\int_{\Omega}^{*} f \mathrm{~d} \mu \leq \int_{\Omega} \tilde{f} \mathrm{~d} \mu=\int_{\Omega} f \mathrm{~d} \mu$ as we can use $\psi=\tilde{f}$ in the definition of the outer integral. Now, in order to prove the reverse inequality $\int_{\Omega} f \mathrm{~d} \mu \leq \int_{\Omega}^{*} f \mathrm{~d} \mu$, we have to consider two cases, depending whether $\int_{\Omega}^{*} f \mathrm{~d} \mu$ is finite or is equal to $-\infty$.

$\diamond$ In the case where $\int_{\Omega}^{*} f \mathrm{~d} \mu$ is finite, we fix $\epsilon>0$. Using Equation (32a), there exists $\psi_{\epsilon} \in L^{1}(\Omega, \mathcal{F}, \mu ; \mathbb{R})$ such that $f \leq \psi_{\epsilon}$ and $\int_{\Omega} \psi_{\epsilon} \mathrm{d} \mu \leq \int_{\Omega}^{*} f \mathrm{~d} \mu+\epsilon$. Using the fact that $f \in L^{1}(\Omega, \mathcal{F}, \mu ; \mathbb{R})$ and the monotonicity of the Lebesgue integral, we obtain

$$
\int_{\Omega} f \mathrm{~d} \mu \leq \int_{\Omega} \psi_{\epsilon} \mathrm{d} \mu \leq \int_{\Omega}^{*} f \mathrm{~d} \mu+\epsilon,
$$

which finally gives $\int_{\Omega} f \mathrm{~d} \mu \leq \int_{\Omega}^{*} f \mathrm{~d} \mu$ and therefore the equality $\int_{\Omega} f \mathrm{~d} \mu=$ $\int_{\Omega}^{*} f \mathrm{~d} \mu$. Equation (34a) follows using Equation (29) as we have

$$
\int_{\Omega}^{*} f \mathrm{~d} \mu=\int_{\Omega} f \mathrm{~d} \mu=\int_{\Omega} f_{+} \mathrm{d} \mu+\left(-\int_{\Omega} f_{-} \mathrm{d} \mu\right)=\int_{\Omega} f_{+} \mathrm{d} \mu \dot{+}\left(-\int_{\Omega} f_{-} \mathrm{d} \mu\right) .
$$

$\diamond$ In the case where $\int_{\Omega}^{*} f \mathrm{~d} \mu=-\infty$, then using Equation (32a) there exists a sequence $\left\{\psi_{n}\right\}_{n \in \mathbb{N}}$ in $L^{1}(\Omega, \mathcal{F}, \mu ; \mathbb{R})$ such that $f \leq \psi_{n}$ and $\int_{\Omega} \psi_{n} \mathrm{~d} \mu \leq-n$ for all $n \in \mathbb{N}$. This implies that $\int_{\Omega} f \mathrm{~d} \mu=-\infty$, which contradicts the fact that $f \in L^{1}(\Omega, \mathcal{F}, \mu ; \mathbb{R})$. 
- Suppose that $\int_{\Omega} f_{+} \mathrm{d} \mu<+\infty$ and $\int_{\Omega} f_{-} \mathrm{d} \mu=+\infty$. Using the fact that $f \leq f_{+}$, we get that $\int_{\Omega}^{*} f \mathrm{~d} \mu \leq \int_{\Omega} f_{+} \mathrm{d} \mu$ as we can use $\psi=f_{+} \in L^{1}(\Omega, \mathcal{F}, \mu ; \mathbb{R})$ in the definition (32a) of the outer integral. Moreover, as $\int_{\Omega} f_{-} \mathrm{d} \mu=+\infty$, we can find a sequence $\left\{\psi_{n}\right\}_{n \in \mathbb{N}}$ of nonnegative functions such that $\psi_{n} \in$ $L^{1}(\Omega, \mathcal{F}, \mu ; \mathbb{R}), \psi_{n} \leq f_{-}$and such that $\lim _{n \rightarrow \infty} \int_{\Omega} \psi_{n} \mathrm{~d} \mu=+\infty$ for all $n \in \mathbb{N}$ (take $\psi_{n}=\mathbf{1}_{\Omega_{n}} \min \left(n, f_{-}\right)$, where $\left(\Omega_{n}\right)_{n \in \mathbb{N}}$ is a monotone sequence of $\mathcal{F}$ measurable subsets of $\Omega$ covering $\Omega$ such that $\mu\left(\Omega_{n}\right)<+\infty$ which exists by $\sigma$-finite property). Using the fact that $\int_{\Omega} f_{+} \mathrm{d} \mu<+\infty$, we can find $\tilde{f} \in L^{1}(\Omega, \mathcal{F}, \mu ; \mathbb{R})$ such that $f_{+}=\tilde{f} \mu$-a.s. Thus, for all $n \in \mathbb{N}$, we have that $f \leq\left(\tilde{f}-\psi_{n}\right)$ and $\left(\tilde{f}-\psi_{n}\right) \in L^{1}(\Omega, \mathcal{F}, \mu ; \mathbb{R})$. We obtain, using monotonicity and monotone convergence that

$$
\int_{\Omega}^{*} f \mathrm{~d} \mu \leq \int_{\Omega}\left(\tilde{f}-\psi_{n}\right) \mathrm{d} \mu=\int_{\Omega} f_{+} \mathrm{d} \mu-\int_{\Omega} \psi_{n} \mathrm{~d} \mu \underset{n \rightarrow+\infty}{\rightarrow}-\infty .
$$

We therefore obtain Equation (34a) since both members of the equality are equal to $-\infty$.

- Suppose that $\int_{\Omega} f_{+} \mathrm{d} \mu=+\infty$ and $\int_{\Omega} f_{-} \mathrm{d} \mu<+\infty$. Then we prove that

$$
\left\{\psi \in L^{1}(\Omega, \mathcal{F}, \mu ; \mathbb{R}) \mid f \leq \psi \quad \mu \text {-a.s. }\right\}=\emptyset .
$$

Indeed, assuming the existence of $\psi \in L^{1}(\Omega, \mathcal{F}, \mu ; \mathbb{R})$ such that $f \leq \psi$, we would obtain that $f_{+} \leq \psi+f_{-}$which, using the fact that $\psi+f_{-} \in$ $L^{1}(\Omega, \mathcal{F}, \mu ; \mathbb{R})$, would imply that $\int_{\Omega} f_{+} \mathrm{d} \mu<+\infty$, hence contradicting the assumption that $\int_{\Omega} f_{+} \mathrm{d} \mu=+\infty$.

- Suppose that $\int_{\Omega} f_{+} \mathrm{d} \mu=+\infty$ and $\int_{\Omega} f_{-} \mathrm{d} \mu=+\infty$. Using the definition of $\dot{+}$, we get that the right hand side of Equation (34a) is equal to $+\infty$. Now, we show that Equation (34a) holds true by proving that the set of functions $\psi \in L^{1}(\Omega, \mathcal{F}, \mu ; \mathbb{R})$ such that $f \leq \psi$ is empty. We proceed by contradiction. Assuming the existence of $\psi \in L^{1}(\Omega, \mathcal{F}, \mu ; \mathbb{R})$ such that $f \leq \psi$, we would have

$$
+\infty=\int_{\Omega} f_{+} \mathrm{d} \mu=\int_{\Omega} f \mathbf{1}_{f \geq 0} \mathrm{~d} \mu \leq \int_{\Omega} \psi \mathbf{1}_{f \geq 0} \mathrm{~d} \mu \leq \int_{\Omega} \psi \mathrm{d} \mu,
$$

contradicting the assumption that $\psi \in L^{1}(\Omega, \mathcal{F}, \mu ; \mathbb{R})$. Therefore, in Equation (32a) we obtain that $\int_{\Omega}^{*} f \mathrm{~d} \mu=+\infty$ and thus equality is ensured in Equation (34a).

This ends the proof. 


\section{References}

\section{References}

[1] M. Akian and E. Fodjo. From a monotone probabilistic scheme to a probabilistic max-plus algorithm for solving Hamilton-Jacobi-Bellman equations. In Hamilton-Jacobi-Bellman equations, volume 21 of Radon Ser. Comput. Appl. Math., pages 1-23. De Gruyter, Berlin, 2018.

[2] C. D. Aliprantis and K. C. Border. Infinite Dimensional Analysis. Springer-Verlag Berlin Heidelberg, New York, 2006.

[3] D. P. Bertsekas and S. E. Shreve. Stochastic Optimal Control: The Discrete Time Case. Optimization and Neural Computation Series. Athena Scientific, Belmont, Mass, 1996.

[4] A. Bourass and E. Giner. Kuhn-Tucker Conditions and Integral Functionals. Journal of Convex Analysis, 8(2):21, 2001.

[5] N. El Karoui and X. Tan. Capacities, Measurable Selection and Dynamic Programming Part I: Abstract Framework. arXiv:1310.3363 [math], page 28, Oct. 2013.

[6] G. Gierz, K. H. Hofmann, K. Keimel, J. D. Lawson, M. Mislove, and D. S. Scott. Continuous Lattices and Domains. Cambridge University Press, first edition, Mar. 2003.

[7] E. Giner. Necessary and Sufficient Conditions for the Interchange Between Infimum and the Symbol of Integration. Set-Valued and Variational Analysis, 17(4):321-357, Dec. 2009.

[8] P. R. Halmos. Measure Theory, volume 18 of Graduate Texts in Mathematics. Springer New York, New York, NY, 1950.

[9] H. Kaise and W. M. McEneaney. Idempotent Expansions for Continuous-Time Stochastic Control. SIAM Journal on Control and Optimization, 54(1):73-98, Jan. 2016.

[10] J. Kawabe. Convergence theorems of the Choquet integral for three types of convergence of measurable functions. Josai Mathematical Monographs, 11:55-74, 2018. 
[11] J. Neveu. Bases Mathématiques Du Calcul Des Probabilités. Masson et Cie, 2 ed. edition, 1970.

[12] A. Pichler and R. Schlotter. Martingale characterizations of risk-averse stochastic optimization problems. Mathematical Programming, 181:377403, 2020.

[13] R. T. Rockafellar and R. J.-B. Wets. Variational Analysis. Number 317 in Die Grundlehren Der Mathematischen Wissenschaften in Einzeldarstellungen. Springer, Dordrecht, corr. 3. print edition, 2009.

[14] A. Shapiro. Interchangeability principle and dynamic equations in risk averse stochastic programming. Operations Research Letters, 45(4):377381, July 2017.

[15] A. Shapiro, D. Dentcheva, and A. P. Ruszczyński. Lectures on Stochastic Programming: Modeling and Theory. Number 9 in MPS-SIAM Series on Optimization. Society for Industrial and Applied Mathematics : Mathematical Programming Society, Philadelphia, 2009. 